\title{
Lower versus higher dose of enteral caloric intake in adult critically ill patients: a systematic review and meta-analysis
}

\author{
Hasan M. Al-Dorzi ${ }^{1,2,3}$, Abdullah Albarrak $^{4}$, Mazen Ferwana ${ }^{1,2,5,6}$, Mohammad Hassan Murad ${ }^{7,8}$ \\ and Yaseen M. Arabi ${ }^{1,2,3^{*}}$
}

\begin{abstract}
Background: There is conflicting evidence about the relationship between the dose of enteral caloric intake and survival in critically ill patients. The objective of this systematic review and meta-analysis is to compare the effect of lower versus higher dose of enteral caloric intake in adult critically ill patients on outcome.

Methods: We reviewed MEDLINE, EMBASE, Cochrane Central Register of Controlled Trials, Cochrane Database of Systematic Reviews, and Scopus from inception through November 2015. We included randomized and quasirandomized studies in which there was a significant difference in the caloric intake in adult critically ill patients, including trials in which caloric restriction was the primary intervention (caloric restriction trials) and those with other interventions (non-caloric restriction trials). Two reviewers independently extracted data on study characteristics, caloric intake, and outcomes with hospital mortality being the primary outcome.
\end{abstract}

Results: Twenty-one trials mostly with moderate bias risk were included (2365 patients in the lower caloric intake group and 2352 patients in the higher caloric group). Lower compared with higher caloric intake was not associated with difference in hospital mortality (risk ratio (RR) 0.953; $95 \%$ confidence interval (Cl) 0.838-1.083), ICU mortality (RR 0. 885; $95 \%$ Cl 0.751-1.042), total nosocomial infections (RR 0.982; $95 \%$ Cl 0.878-1.077), mechanical ventilation duration, or length of ICU or hospital stay. Blood stream infections (11 trials; RR 0.718; $95 \% \mathrm{Cl} 0.519-0.994$ ) and incident renal replacement therapy (five trials; RR 0.711; $95 \%$ Cl 0.545-0.928) were lower with lower caloric intake. The associations between lower compared with higher caloric intake and primary and secondary outcomes, including pneumonia, were not different between caloric restriction and non-caloric restriction trials, except for the hospital stay which was longer with lower caloric intake in the caloric restriction trials.

Conclusions: We found no association between the dose of caloric intake in adult critically ill patients and hospital mortality. Lower caloric intake was associated with lower risk of blood stream infections and incident renal replacement therapy (five trials only). The heterogeneity in the design, feeding route and timing and caloric dose among the included trials could limit our interpretation. Further studies are needed to clarify our findings.

Keywords: Enteral feeding, Nutrition, Intensive care unit, Cross infection, Mortality

\footnotetext{
* Correspondence: yaseenarabi@yahoo.com; icu1@ngha.med.sa

${ }^{1}$ College of Medicine, King Saud bin Abdulaziz University for Health Sciences, Riyadh, Saudi Arabia

${ }^{2}$ King Abdullah International Medical Research Center, Riyadh, Saudi Arabia

Full list of author information is available at the end of the article
} 


\section{Background}

Nutritional support is essential in the management of adult critically ill patients $[1,2]$. Supported by randomized controlled trials (RCTs) and systematic reviews [2], early initiation of enteral nutrition (EN) has been shown to be associated with better outcome compared to late EN. However, it is less clear what the most appropriate caloric dose is. Based on expert opinion, observational studies and small RCTs [3-7], it has been generally recommended to provide full caloric requirement to critically ill patients [8]. To achieve this goal, interventions to augment caloric intake, such as the implementation of protocols [9, 10], prokineteic agents and postpyloric tube placement [11] have been proposed, even though studies have not demonstrated improvement in clinical outcomes. On the contrary, several observational studies observed better outcome with lower enteral caloric intake $[12,13]$.

These conflicting results may be related to differences in study populations, selection bias and immortal time bias (nutritional intake is better for patients who survive and have a longer ICU stay). Prescribed hypocaloric nutrition has recently been tested in several randomized controlled studies [14-16]. Although parenteral nutrition (PN) differs from EN in indications, physiologic effects and complications, studies have shown that lower caloric intake with PN was associated with better clinical outcomes [17]. As such the clinical practice guidelines of the Society of Critical Care Medicine and American Society for Parenteral and Enteral Nutrition recommended that mild permissive underfeeding should be considered in critically ill patients receiving $\mathrm{PN}$, at least in certain highrisk groups $[8,18]$. On the other hand, the evidence on the relationship between enteral caloric intake and survival remains conflicting and has ignited heated discussion in the critical care literature $[19,20]$.

Given the present controversies, a systematic review that includes RCTs is likely to produce more reliable effect estimates. Therefore, we conducted this systematic review and meta-analysis to compare the impact of lower versus higher dose of enteral caloric intake in adult critically ill patients on mortality and other important outcomes.

\section{Methods}

This systematic review is reported according to the statement of Preferred Reporting Items for Systematic Reviews and Meta-analyses (PRISMA) [21].

\section{Literature search}

We conducted a comprehensive search of several databases from the earliest inception of each database to November 2015 for randomized trials examining the effect of EN dose on the outcomes of critically ill patients. The databases included Ovid Medline In-Process and Other Non-Indexed Citations, Ovid MEDLINE, Ovid EMBASE,
Ovid Cochrane Central Register of Controlled Trials, Ovid Cochrane Database of Systematic Reviews, and Scopus. The search strategy was designed and conducted by an experienced librarian. The detailed search strategy including that of Scopus is presented in Additional file 1.

We also conducted a manual search of the bibliographies of all selected articles, systematic reviews on nutritional support in critically ill patients and studies published as abstracts in the preceding five meetings of the American Thoracic Society (2010-2015), Society of Critical Care Medicine (2010-2015), American College of Chest Physicians (2010-2015), American Society for Parenteral and Enteral Nutrition (2010-2015) and European Society for Parenteral and Enteral Nutrition (2010-2015).

\section{Study selection}

Two authors (YA and HD) independently assessed the titles and abstracts from the search results for eligibility. We included studies that met the following criteria: (1) randomized or quasi-randomized design; (2) enrolled adults who were critically ill and required care in an ICU setting; (3) primarily compared two doses of EN; (4) reported caloric intake either in absolute values (i.e., in kcal) or in percentage of caloric requirement as defined by authors; and (5) had a meaningful difference in caloric intake between the two groups (statistically significant or if the difference was $\geq 10 \%$ ). The $10 \%$ difference was defined a priori by the authors of this review as the minimally significant difference.

We excluded studies: (1) of PN as a primary intervention; (2) that compared early versus late EN; (3) that assessed enteral formulae that had immune-modulating ingredients; (4) that evaluated postpyloric placement of the feeding tube for gastrointestinal reasons (such as pancreatitis) and not primarily to increase caloric intake; (5) that were cluster randomized or crossover studies; (6) that were trials with only surrogate outcomes (such as nutritional, biochemical, economic or quality of life assessment endpoints); and (7) that were trials published in abstract form only or in a non-English language.

The included trials in this systematic review fell in one of two categories. The first category was studies that had caloric restriction as the primary intervention defined as deliberate reduction of caloric intake from the estimated requirement. In these studies, which we refer to in the rest of the paper as caloric restriction trials, the lower dose included trophic feeding (minimal amounts of calories, i.e., $20 \mathrm{kcal} / \mathrm{h}$ ) $[15,16]$ and hypocaloric feeding or permissive underfeeding (moderate amount of calories, i.e., close to $50 \%$ of caloric requirement) [14, 22, 23]. In these trials, the higher dose was standard or eucaloric feeding (usually $70-100 \%$ of caloric need) [14-16, 22, 23]. The second category, which we referred to as non-caloric restriction trials, included studies in which an intervention 
was tested that led directly or indirectly to change in caloric intake. These interventions included, but were not limited to, protocol implementation, postpyloric tube placement and the use of prokinetics. We resolved differences by discussion. All potentially eligible studies were retrieved in full.

\section{Risk of bias assessment}

Two reviewers (HD and YA) independently assessed the methodological quality of each trial using the Cochrane Collaboration tool for Assessing Risk of Bias [24]. Any discrepancies between the two reviewers were resolved through discussion. The measure of agreement between reviewers was calculated using kappa statistics.

\section{Data extraction}

Two non-blinded reviewers (HD and $\mathrm{AB}$ ) independently abstracted pertinent data from the trials using a standardized predefined form. Extracted data included study design, study size, study setting, patient population, reported illness severity score, interventions and their duration and caloric intake (mean and percentage of estimated caloric target) in each arm. The primary outcome of this review was all-cause hospital mortality. Other important outcomes were chosen a priori and included ICU mortality, bloodstream infection as defined by the authors, pneumonia as defined by the authors, all infections, incident or new renal replacement therapy, mechanical ventilation duration and ICU and hospital length of stay (LOS). For published reports with insufficient information, we attempted to contact the corresponding author for clarification. Two authors replied and the two studies were later excluded.

\section{Statistical analysis}

From each study, we extracted the number of events and sample size in each arm (binary outcomes) and the mean with a measure of variability (continuous outcomes). Metaanalysis was performed using the random-effects model as described by DerSimonian and Laird [25]. The pooledeffect estimates of lower versus higher caloric intake were reported as risk ratio (RR) and weighted mean difference (WMD) with $95 \%$ confidence intervals (CIs).

We performed subgroup analyses with stratification by caloric restriction versus non-caloric-restriction trials which had mean patients' age $<65$ versus those with mean age $\geq 65$ years, trials which had mean Acute Physiology and Chronic Health Evaluation (APACHE) II score $<20$ versus those with mean APACHE II $\geq 20$, trials in which the lower calorie group received $<60 \%$ versus those in which the lower calorie group received $\geq 60 \%$ of requirement and trials in which the calorie difference between the two groups $<20 \%$ versus those with the difference $\geq 20 \%$. We tested for interaction between the subgroups and considered the interaction test to be significant when its $p$ value was $<0.05$. We also conducted meta-regression to assess the effect of the difference in caloric intake between the arms in each study and of the amount of provided calories in the lower calorie group in each study on hospital mortality.

All statistical tests were two-sided and a $p$ value $<0.05$ was considered statistically significant. The degree of heterogeneity among the studies was assessed by the $I^{2}$ statistic [26]. Between-study heterogeneity was considered low if $I^{2}$ was $<30 \%$ whereas $I^{2}>50 \%$ represented substantial heterogeneity. A small study bias or publication bias was assessed by visual inspection of the funnel plot and by conducting the Egger regression test. We used the Comprehensive Meta analysis Software (Version 3, Englewood, NJ, USA) for all analyses.

\section{Results}

Figure 1 shows the flow chart of the study selection process. Through the electronic database search, we identified 449 citations. We excluded 413 studies after screening the titles and abstracts. The manual search of abstracts from conferences and of bibliographies yielded 12 additional studies that met the inclusion criteria. The full texts of 50 studies were evaluated and 29 studies were excluded $[9,10,27-53]$ for different reasons as shown in Fig. 1 . Twenty-one studies were eligible for data extraction and analysis [14-16, 22, 23, 54-69]. We followed the intentionto-treat principle whenever possible.

\section{Description of included studies}

A summary of the included studies is presented in Table 1 . The total sample size was 4717 patients $(2365$ in the lower caloric intake group and 2352 in the high caloric group). Seven studies [14-16, 22, 23, 68, 69] directly compared caloric restriction provided by enteral tube feeding with standard feeding and 14 assessed the effect of an intervention that led to a significant difference in caloric intake. The mean difference in caloric intake was $445 \mathrm{kcal}$ (range 165-1118 kcal). The difference in calories between the intervention groups corresponded to $14-78.8 \%$ of the higher caloric intake.

Table 2 describes the risk of bias assessment of the studies and shows that most studies had bias risk in at least one domain of the Cochrane Collaboration tool. All except two studies $[57,66]$ were un-blinded. Agreement between reviewers (kappa) on the tool elements was 0.774 (95\% CI 0.651-0.892; $p<0.001$ ).

\section{Effect on mortality}

Nineteen studies provided data on hospital mortality [14-16, 22, 54, 55, 57-69]. Figure 2a describes the corresponding forest plot. Lower compared with higher caloric intake was not associated with difference in hospital mortality; the overall RR was 0.953 (95\% CI, $0.838-$ 


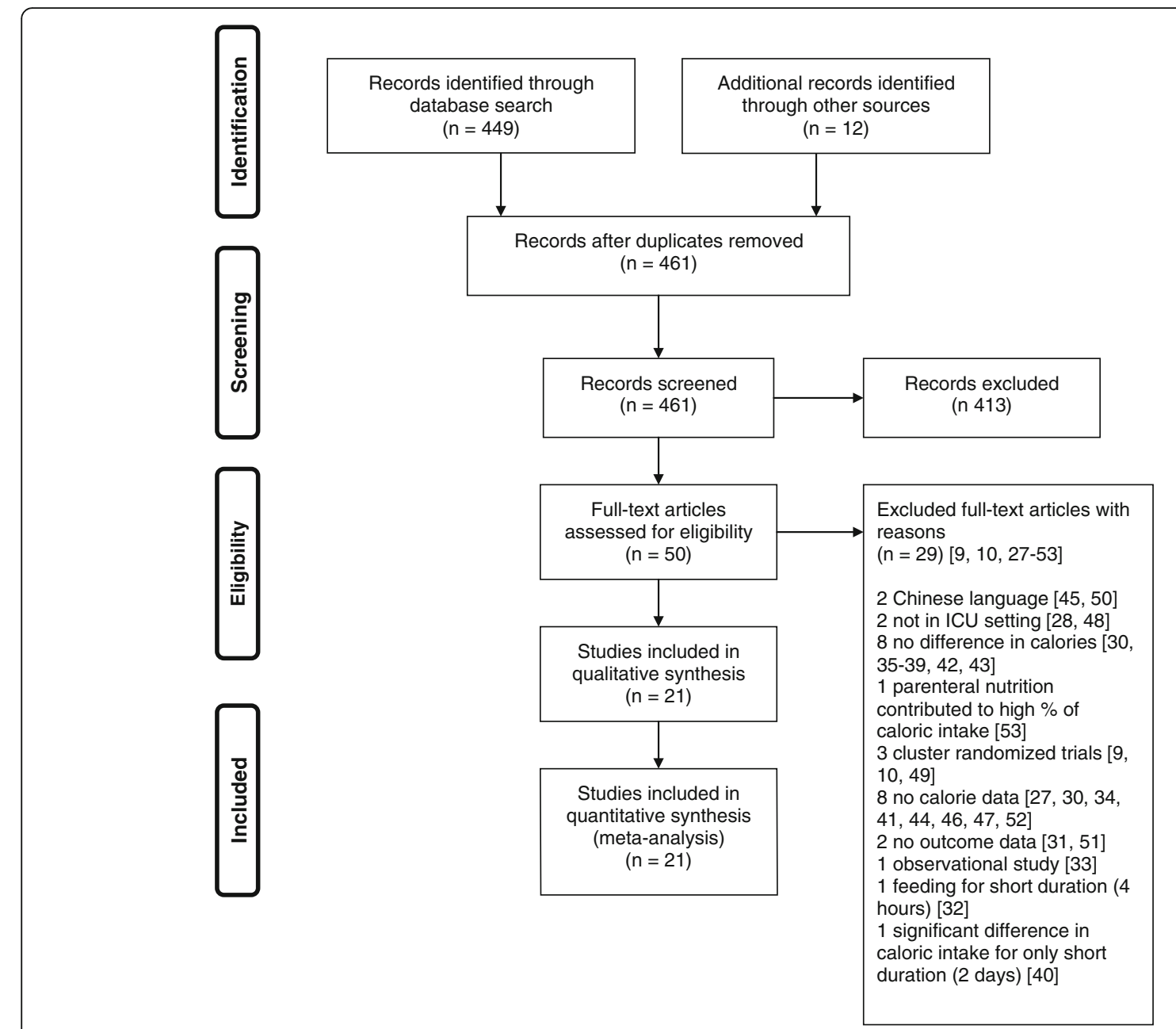

Fig. 1 Preferred Reporting Items for Systematic Reviews and Meta-analyses (PRISMA) flow diagram

1.083). The association between lower compared with higher caloric intake and hospital mortality was not different between caloric restriction [14-16, 22, 68, 69] and non-caloric restriction trials [54, 55, 57-67] (interaction test $p=0.19)$.

Similarly, the association between lower compared with higher caloric intake and hospital mortality was not different between studies in which the mean age of patients was $\geq 65$ years $[59,64]$ and those in which the mean age was $<65$ years $[14-16,22,54,55,57,58,60-63$, 65-69] (RR 0.994; 95 \% CI 0.825-1.079; interaction test $p=0.54$ ), between studies in which the mean APACHE II score was $<20[22,61,62,69]$, and those in which the mean APACHE II score was $\geq 20[14,15,54,55,57,59$, 60, 63, 64, 66-68] (RR 0.977; 95 \% CI 0.862-1.108; interaction test $p=0.73$ ), between studies [14-16, 22, $54,68,69]$ where the lower calorie group received $<60 \%$ of requirement and studies where the lower calorie group received $\geq 60 \%[55,57-67]$ (RR 0.946;
$95 \%$ CI 0.832-1.075; interaction test $p=0.20$ ) and between the studies in which the calorie difference between the two groups $\geq 20 \%[15,16,22,55,66-69]$ and those with calorie difference $<20 \%[14,58,60,62,64]$ (RR 0.977; $95 \% \mathrm{CI}$ $0.860-1.110$; interaction test $p=0.97)$.

The meta-regression analysis (Fig. 2b and c) showed that hospital mortality was not influenced by the difference in caloric intake between the lower and higher calorie groups in each trial (slope $=-0.0002 ; p=0.56$ ) or by the lower caloric dose in each trial (slope $=0.0001 ; p=0.69$ ). Additionally, there was low heterogeneity among the studies $\left(I^{2}=26.4 \%\right)$ and no evidence of publication bias on inspection of the funnel plot (Fig. 2d), with $p=0.79$ for Egger's test.

Eight studies had data on ICU mortality. Figure 3 presents the corresponding forest plot. The overall RR was 0.885 (95\% CI 0.751-1.042). There was no significant difference between caloric restriction and non-caloricrestriction trials (interaction test $p=0.32$ ). There was no 
Table 1 Characteristics of studies included in the systematic review

\begin{tabular}{|c|c|c|c|c|c|c|c|c|c|c|}
\hline Author, year & Population & Design & $\begin{array}{l}\text { Number of } \\
\text { patients }\end{array}$ & Age (mean) & Male (\%) & APACHE II (mean) & MV & $\begin{array}{l}\text { Intervention (daily } \\
\text { caloric intake) }\end{array}$ & $\begin{array}{l}\text { Control (daily } \\
\text { caloric intake) }\end{array}$ & $\begin{array}{l}\text { Duration of } \\
\text { intervention }\end{array}$ \\
\hline $\begin{array}{l}\text { Montecalvo et al., } \\
1992 \text { [54] }^{\mathrm{a}}\end{array}$ & $\begin{array}{l}\text { Medical and } \\
\text { surgical }\end{array}$ & $\mathrm{RCT}$ & 38 & $\begin{array}{l}50.5 \text { years in the } \\
\text { intervention } \\
\text { group, } 44.8 \\
\text { years in the } \\
\text { control group }\end{array}$ & 60.5 & $\begin{array}{l}\text { Acute physiology } \\
\text { score of APACHE ॥ } \\
24.0 \text { in the } \\
\text { intervention group, } \\
21.7 \text { in the control } \\
\text { group }\end{array}$ & $\begin{array}{l}\text { Not reported } \\
\text { Probably all }\end{array}$ & $\begin{array}{l}\text { Jejunal feeding } \\
(1182 \pm 603 \mathrm{kcal} ; \\
46.9 \pm 25.9 \% \text { of goal })\end{array}$ & $\begin{array}{l}\text { Gastric feeding } \\
(1466 \pm 398 \mathrm{kcal} ; \\
61.0 \pm 17 \% \text { of } \\
\text { goal })\end{array}$ & $\begin{array}{l}\text { As long as tube } \\
\text { feeding was } \\
\text { required Mean } \\
\text { tube feeding } \\
\text { was } 10 \text { days }\end{array}$ \\
\hline $\begin{array}{l}\text { Kearns et al., } \\
2000[55]\end{array}$ & Medical & $\mathrm{RCT}$ & 44 & $\begin{array}{l}54 \text { years in the } \\
\text { intervention } \\
\text { group, } 49 \\
\text { years in the } \\
\text { control group }\end{array}$ & 68 & $\begin{array}{l}22 \text { in the intervention } \\
\text { group, } 20 \text { in the } \\
\text { control group }\end{array}$ & $100 \%$ & $\begin{array}{l}\text { Small intestinal feeding } \\
(1157 \pm 86 \mathrm{kcal} ; 18 \pm \\
1 \mathrm{kcal} / \mathrm{kg} / \text { day; } 69 \pm 7 \% \\
\text { of caloric requirement) } \\
\text { Protein intake: } 0.7 \pm \\
0.1 \mathrm{~g} / \mathrm{kg} / \mathrm{kg} / \text { day }\end{array}$ & $\begin{array}{l}\text { Gastric feeding } \\
(812 \pm 122 \mathrm{kcal} ; \\
12 \pm 2 \mathrm{kcal} / \mathrm{kg} / \mathrm{day} ; \\
47 \pm 7 \% \text { of caloric } \\
\text { requirement). } \\
\text { Protein intake } \\
0.4 \pm 0.1 \mathrm{~g} / \mathrm{kg} / \mathrm{kg} / \mathrm{day}\end{array}$ & $7-10$ days \\
\hline $\begin{array}{l}\text { Chen et al., } \\
2006 \text { [56] }]^{\mathrm{a}}\end{array}$ & Medical & $\mathrm{RCT}$ & 107 & $\begin{array}{l}\text { Similar age } \\
\text { distribution in } \\
\text { the control and } \\
\text { intervention } \\
\text { groups }\end{array}$ & 76.6 & $\begin{array}{l}\text { Similar APACHE II } \\
\text { distribution in both } \\
\text { groups }\end{array}$ & $100 \%$ & $\begin{array}{l}\text { Continuous feeding, } \\
\text { with calories significantly } \\
\text { different from the other } \\
\text { group }\end{array}$ & Intermittent feeding & $\begin{array}{l}\text { Not clear At } \\
\text { least } 7 \text { days }\end{array}$ \\
\hline $\begin{array}{l}\text { Nguyen et al., } \\
2007[57]^{a}\end{array}$ & Medical & $\mathrm{RCT}$ & 75 & $\begin{array}{l}50.9 \text { years in the } \\
\text { intervention } \\
\text { group, } 52 \text { years } \\
\text { in the control } \\
\text { group }\end{array}$ & 70 & $\begin{array}{l}23 \text { in the intervention } \\
\text { group, } 22.6 \text { in the } \\
\text { control group }\end{array}$ & $100 \%$ & $\begin{array}{l}\text { Combination prokinetic } \\
\text { therapy (erythromycin } \\
\text { and metoclopramide) } \\
\text { Significantly higher } \\
\text { caloric intake }\end{array}$ & $\begin{array}{l}\text { Erythromycin alone } \\
\text { Lower caloric intake }\end{array}$ & 7 days \\
\hline $\begin{array}{l}\text { Desachy et al., } \\
2008[58]^{a}\end{array}$ & $\begin{array}{l}\text { Medical and } \\
\text { surgical }\end{array}$ & RCT & 100 & $\begin{array}{l}64 \text { years in the } \\
\text { interventional } \\
\text { group, } 58 \text { years } \\
\text { in the control } \\
\text { group }\end{array}$ & 69 & $\begin{array}{l}\text { APACHE II is not } \\
\text { reported SAPS II: } 40 \\
\text { in gradual feeding } \\
\text { group, } 42 \text { in } \\
\text { immediate feeding } \\
\text { group }\end{array}$ & $100 \%$ & $\begin{array}{l}\text { Gradual feeding } 76 \% \\
\text { of optimal calorie } \\
\text { intake (1297 } \pm 331 \mathrm{kcal})\end{array}$ & $\begin{array}{l}\text { Immediate feeding } \\
95 \% \text { of optimal } \\
\text { calorie intake } \\
(1715 \pm 331 \mathrm{kcal})\end{array}$ & $\begin{array}{l}120 \pm 48 \text { hours } \\
\text { (similar in the } \\
\text { two groups); } \\
45 \text { patients } \\
\text { were followed } \\
\text { for maximum } \\
\text { of } 7 \text { days }\end{array}$ \\
\hline $\begin{array}{l}\text { Hsu et al., } \\
2009 \text { [59] }\end{array}$ & Medical & $\mathrm{RCT}$ & 121 & $\begin{array}{l}70 \text { years in the } \\
\text { intervention, } \\
67.9 \text { years in the } \\
\text { control group }\end{array}$ & 70.2 & $\begin{array}{l}20.5 \text { in the } \\
\text { intervention group, } \\
20.3 \text { in the control } \\
\text { group }\end{array}$ & $100 \%$ & $\begin{array}{l}\text { Nasoduodenal feeding } \\
\text { group (1658 } 118 \mathrm{kcal} ; \\
27.1 \pm 7.6 \mathrm{kcal} / \mathrm{kg} / \mathrm{day}) \\
\text { Protein intake: } 1.11 \pm \\
0.31 \mathrm{~g} / \mathrm{kg} / \text { day }\end{array}$ & $\begin{array}{l}\text { Nasogastric feeding } \\
\text { group }(1426 \pm \\
110 \mathrm{kcal} ; 23.5 \pm \\
8.8 \mathrm{kcal} / \mathrm{kg} / \text { day }) \\
\text { Protein intake } 0.97 \pm \\
0.39 \mathrm{~g} / \mathrm{kg} / \text { day }\end{array}$ & $\begin{array}{l}\text { Mean study } \\
\text { period was } \\
11 \text { days }\end{array}$ \\
\hline $\begin{array}{l}\text { White et al., } \\
2009 \text { [60] }\end{array}$ & Medical & $\mathrm{RCT}$ & 104 & $\begin{array}{l}50 \text { years in the } \\
\text { intervention } \\
\text { group, } 54 \text { years } \\
\text { in the control } \\
\text { group }\end{array}$ & 50 & $\begin{array}{l}30 \text { in the intervention } \\
\text { group, } 24.5 \text { in the } \\
\text { control group }\end{array}$ & $100 \%$ & $\begin{array}{l}\text { Postpyloric feeding } \\
\text { (1296 kcal; } 88.5 \% \text { of } \\
\text { caloric requirement) } \\
\text { Average protein } \\
\text { deficit } 6.5 \mathrm{~g} / \text { day }\end{array}$ & $\begin{array}{l}\text { Gastric feeding } \\
\text { (1515 kcal; } 95 \% \text { of } \\
\text { caloric requirement) } \\
\text { Average protein } \\
\text { deficit } 3.5 \mathrm{~g} / \text { day }\end{array}$ & $\begin{array}{l}\text { Not reported } \\
\text { Mean gastric } \\
\text { feeding duration } \\
\text { was } 3.1 \text { days, } \\
\text { mean postpyloric } \\
\text { feeding duration } \\
\text { was } 4.0 \text { days }\end{array}$ \\
\hline $\begin{array}{l}\text { Montejo et al., } \\
2010[61]^{a}\end{array}$ & $\begin{array}{l}\text { Medical and } \\
\text { surgical }\end{array}$ & RCT & 329 & $\begin{array}{l}65 \text { years in the } \\
\text { intervention } \\
\text { group, } 60 \text { years }\end{array}$ & 65 & $\begin{array}{l}19.4 \text { in the } \\
\text { intervention group, }\end{array}$ & $100 \%$ & $\begin{array}{l}\text { High gastric residual } \\
\text { volume of } 500 \mathrm{ml}\end{array}$ & $\begin{array}{l}\text { Low gastric residual } \\
\text { volume of } 200 \mathrm{ml} \\
\text { (diet volume ratio }\end{array}$ & $\begin{array}{l}\text { The duration of } \\
\text { enteral nutrition }\end{array}$ \\
\hline
\end{tabular}


Table 1 Characteristics of studies included in the systematic review (Continued)

\begin{tabular}{|c|c|c|c|c|c|c|c|c|c|c|}
\hline & & & & $\begin{array}{l}\text { in the control } \\
\text { group }\end{array}$ & & $\begin{array}{l}18.9 \text { in the control } \\
\text { group }\end{array}$ & & $\begin{array}{l}\text { (diet volume ratio in } \\
\text { the first week }=88.2 \% \text { ) }\end{array}$ & $\begin{array}{l}\text { in the first week = } \\
84.5 \%)\end{array}$ & $\begin{array}{l}\text { (maximum of } \\
28 \text { days) }\end{array}$ \\
\hline $\begin{array}{l}\text { Acosta-Escribano } \\
\text { et al., } 2010[62]^{a}\end{array}$ & $\begin{array}{l}\text { Severe traumatic } \\
\text { brain injury }\end{array}$ & RCT & 104 & $\begin{array}{l}35 \text { years in the } \\
\text { intervention } \\
\text { group, } 41 \text { years } \\
\text { in the control } \\
\text { group }\end{array}$ & 86.5 & $\begin{array}{l}16 \text { in the intervention } \\
\text { group, } 18 \text { in the } \\
\text { control group }\end{array}$ & $100 \%$ & $\begin{array}{l}\text { Transpyloric feeding } \\
\text { ( } 92 \% \text { of the feeding } \\
\text { volume given) }\end{array}$ & $\begin{array}{l}\text { Gastric feeding } \\
\text { ( } 84 \% \text { of the } \\
\text { feeding volume } \\
\text { given) }\end{array}$ & Not reported \\
\hline $\begin{array}{l}\text { Arabi et al., } \\
2011 \text { [14] }\end{array}$ & $\begin{array}{l}\text { Medical and } \\
\text { surgical }\end{array}$ & RCT & 240 & $\begin{array}{l}50.3 \text { years in the } \\
\text { intervention } \\
\text { group, } 5.19 \text { years } \\
\text { in the control } \\
\text { group }\end{array}$ & 68.3 & $\begin{array}{l}25.2 \text { in the } \\
\text { intervention group, } \\
25.3 \text { in the control } \\
\text { group }\end{array}$ & $99.2 \%$ & $\begin{array}{l}\text { Permissive under } \\
\text { feeding: } 59 \% \text { of } \\
\text { requirement }(1066 \pm \\
306 \mathrm{kcal} ; \\
13.9 \mathrm{kcal} / \mathrm{kg} / \text { day }) \\
\text { Protein intake } \\
0.61 \mathrm{~g} / \mathrm{kg} / \text { day }\end{array}$ & $\begin{array}{l}\text { Target feeding } \\
71.4 \% \text { of } \\
\text { requirement } \\
(1251 \pm 432 \mathrm{kcal} ; \\
16.4 \mathrm{kcal} / \mathrm{kg} / \mathrm{day}) \\
\text { Protein intake } \\
0.57 \mathrm{~g} / \mathrm{kg} / \text { day }\end{array}$ & $\begin{array}{l}\text { Duration of } \\
\text { enteral feeding } \\
\text { or ICU discharge }\end{array}$ \\
\hline $\begin{array}{l}\text { Singer et al., } \\
2011 \text { [63] }\end{array}$ & $\begin{array}{l}\text { Medical, surgical } \\
\text { and trauma }\end{array}$ & $\mathrm{RCT}$ & 130 & $\begin{array}{l}59 \text { years in the } \\
\text { intervention } \\
\text { group, } 62 \text { years } \\
\text { in the control } \\
\text { group }\end{array}$ & 58 & $\begin{array}{l}22.1 \text { in the } \\
\text { intervention group, } \\
22.4 \text { in the control } \\
\text { group }\end{array}$ & $100 \%$ & $\begin{array}{l}\text { Tight caloric intake } \\
\text { according to } \\
\text { indirect calorimetry } \\
\text { (2086 } \pm 467 \text { kcal) } \\
\text { Protein intake } \\
0.95 \mathrm{~g} / \mathrm{kg} / \text { day }\end{array}$ & $\begin{array}{l}\text { Standard caloric } \\
\text { intake at } \\
25 \mathrm{kcal} / \mathrm{kg} / \text { day } \\
(1480 \pm 356 \mathrm{kcal}) \\
\text { Protein intake } \\
0.68 \mathrm{~g} / \mathrm{kg} / \text { day }\end{array}$ & $\begin{array}{l}\text { Not clear. Till } \\
\text { day } 14 \text { or } \\
\text { discharge } \\
\text { from the ICU }\end{array}$ \\
\hline $\begin{array}{l}\text { Rice et al., } \\
2011 \text { [15] }\end{array}$ & $\begin{array}{l}\text { Acute respiratory } \\
\text { failure }\end{array}$ & $\mathrm{RCT}$ & 200 & $\begin{array}{l}53 \text { years in the } \\
\text { intervention } \\
\text { group, } 54 \text { years } \\
\text { in the control } \\
\text { group }\end{array}$ & 44 & 26.9 in both groups & $100 \%$ & $\begin{array}{l}\text { Trophic feeding } \\
(300 \pm 149 \mathrm{kcal} ; \\
15.8 \pm 11 \% \text { of } \\
\text { caloric requirement }) \\
\text { Protein intake: } \\
0.13 \mathrm{~g} / \mathrm{kg} / \text { day }\end{array}$ & $\begin{array}{l}\text { Full feeding } \\
(1418 \pm 686 \mathrm{kcal} ; \\
74.8 \pm 38.5 \% \text { of } \\
\text { caloric requirement }) \\
\text { Protein intake } \\
0.66 \mathrm{~g} / \mathrm{kg} / \text { day }\end{array}$ & 6 days \\
\hline $\begin{array}{l}\text { Rice et al., } \\
2012[16]^{a}\end{array}$ & $\begin{array}{l}\text { ARDS patients } \\
\text { (medical, surgical } \\
\text { and trauma) }\end{array}$ & RCT & 1000 & $\begin{array}{l}52 \text { years in the } \\
\text { intervention } \\
\text { and control } \\
\text { groups }\end{array}$ & 51 & $\begin{array}{l}\text { APACHE III: } 92 \text { in the } \\
\text { intervention group, } \\
90 \text { in the control } \\
\text { group }\end{array}$ & $100 \%$ & $\begin{array}{l}\text { Trophic feeding } \\
\text { ( } 400 \mathrm{kcal} ; 25 \% \text { of } \\
\text { estimated non- } \\
\text { protein caloric } \\
\text { requirement) }\end{array}$ & $\begin{array}{l}\text { Full feeding } \\
\text { (1300 kcal; } 80 \% \\
\text { of estimated caloric } \\
\text { requirement) }\end{array}$ & 6 days \\
\hline $\begin{array}{l}\text { Huang et al., } \\
2012 \text { [64] }\end{array}$ & Medical & $\mathrm{RCT}$ & 101 & $\begin{array}{l}70.9 \text { years in the } \\
\text { intervention } \\
\text { group, } 68.3 \text { years } \\
\text { in the control } \\
\text { group }\end{array}$ & 71 & $\begin{array}{l}21.0 \text { in the } \\
\text { intervention group, } \\
19.6 \text { in the control } \\
\text { group }\end{array}$ & $100 \%$ & $\begin{array}{l}\text { Nasoduodenal } \\
\text { feeding (1575 kcal; } \\
90.4 \% \text { of target } \\
\text { energy intake). } \\
\text { Protein intake } 93.2 \pm \\
26.9 \% \text { of target }\end{array}$ & $\begin{array}{l}\text { Nasogastric feeding } \\
\text { (1343 kcal; } 76.2 \% \text { of } \\
\text { target energy intake) } \\
\text { Protein intake } 78.6 \pm \\
28.5 \% \text { of target }\end{array}$ & 21 days \\
\hline $\begin{array}{l}\text { Reignier et al., } \\
2013[65]^{a}\end{array}$ & $\begin{array}{l}\text { Medical and } \\
\text { surgical }\end{array}$ & $\mathrm{RCT}$ & 449 & $\begin{array}{l}61 \text { years in the } \\
\text { intervention } \\
\text { group, } 62 \text { years } \\
\text { in the control } \\
\text { group }\end{array}$ & 70 & $\begin{array}{l}\text { Baseline SOFA } 8 \text { for } \\
\text { both groups }\end{array}$ & $100 \%$ & $\begin{array}{l}\text { Not monitoring } \\
\text { residual gastric } \\
\text { volume (calorie } \\
\text { deficit } 319 \text { kcal) }\end{array}$ & $\begin{array}{l}\text { Monitoring residual } \\
\text { gastric volume } \\
\text { (calorie deficit } \\
509 \text { kcal) }\end{array}$ & $\begin{array}{l}\text { Not clear } \\
\text { Follow up } \\
\text { for } 90 \text { days }\end{array}$ \\
\hline $\begin{array}{l}\text { Rugeles et al., } \\
2013 \text { [23] }\end{array}$ & $\begin{array}{l}\text { Medical and } \\
\text { surgical }\end{array}$ & $\mathrm{RCT}$ & 80 & $\begin{array}{l}53.3 \text { years in the } \\
\text { intervention } \\
\text { group, } 55.7 \text { years } \\
\text { in the control } \\
\text { group }\end{array}$ & 58 & $\begin{array}{l}13.9 \text { in the } \\
\text { intervention group, } \\
15.1 \text { in the control } \\
\text { group }\end{array}$ & & $\begin{array}{l}\text { Hyperproteic } \\
\text { hypocaloric enteral } \\
\text { nutrition as } \\
15 \mathrm{kcal} / \mathrm{kg} / \text { day }\end{array}$ & $\begin{array}{l}\text { Standard nutritional } \\
\text { regimen as } \\
25 \mathrm{kcal} / \mathrm{kg} / \text { day } \\
(921 \mathrm{kcal}) \text { Protein } \\
\text { intake } 0.76 \mathrm{~g} / \mathrm{kg} / \text { day }\end{array}$ & 7 days \\
\hline
\end{tabular}


Table 1 Characteristics of studies included in the systematic review (Continued)

\begin{tabular}{|c|c|c|c|c|c|c|c|c|c|c|}
\hline & & & & & & & & $\begin{array}{l}\text { (756 kcal) Protein } \\
\text { intake } 1.4 \mathrm{~g} / \mathrm{kg} / \text { day }\end{array}$ & & \\
\hline $\begin{array}{l}\text { Peake et al., } \\
2014 \text { [66] }\end{array}$ & $\begin{array}{l}\text { Mechanically } \\
\text { ventilated } \\
\text { (medical and } \\
\text { surgical) }\end{array}$ & RCT & 112 & $\begin{array}{l}56.4 \text { years in the } \\
\text { intervention } \\
\text { group, } 56.5 \text { years } \\
\text { in the control } \\
\text { group }\end{array}$ & 74 & $\begin{array}{l}23 \text { in the intervention } \\
\text { group, } 22 \text { in the } \\
\text { control group }\end{array}$ & $100 \%$ & $\begin{array}{l}\text { Nutritional formula } \\
1.5 \mathrm{kcal} / \mathrm{ml}(1832 \pm \\
381 \mathrm{kcal} ; 27.3 \pm \\
7.4 \mathrm{kcal} / \mathrm{kg} ; 96.0 \% \\
\text { of requirement) } \\
\text { Protein intake } \\
75 \% \text { of target }\end{array}$ & $\begin{array}{l}\text { Nutritional formula } \\
1 \mathrm{kcal} / \mathrm{ml}(1259 \pm \\
428 \mathrm{kcal} ; 19.0 \pm \\
6.0 \mathrm{kcal} / \mathrm{kg} ; 68.4 \% \\
\text { of requirement) } \\
\text { Protein intake } 79 \% \\
\text { of target }\end{array}$ & 10 days \\
\hline $\begin{array}{l}\text { Charles et al., } \\
2014 \text { [22] }\end{array}$ & Surgical/ trauma & $\mathrm{RCT}$ & 83 & $\begin{array}{l}50.4 \text { years in the } \\
\text { intervention } \\
\text { group, } 53.4 \text { years } \\
\text { in the control } \\
\text { group }\end{array}$ & 71 & $\begin{array}{l}16.6 \text { in the } \\
\text { intervention group, } \\
17.3 \text { in the control } \\
\text { group }\end{array}$ & $62.7 \%$ & $\begin{array}{l}\text { Hypocaloric feeding: } \\
50 \% \text { of estimated } \\
\text { requirement as } 12.5- \\
15 \mathrm{kcal} / \mathrm{kg} / \text { day } \\
(982 \pm 61 \mathrm{kcal} ; \\
12.3 \pm 0.7 \mathrm{kcal} / \mathrm{kg} / \text { day }) \\
\text { Protein intake } \\
1.1 \mathrm{~g} / \mathrm{kg} / \text { day }\end{array}$ & $\begin{array}{l}\text { Eucaloric feeding } \\
100 \% \text { of estimated } \\
\text { requirement as } \\
25-30 \mathrm{kcal} / \mathrm{kg} / \text { day } \\
(1338 \pm 92 \mathrm{kcal} ; \\
17.1 \pm 1.1 \mathrm{kcal} / \mathrm{kg} / \text { day }) \\
\text { Protein intake } \\
1.1 \mathrm{~g} / \mathrm{kg} / \text { day }\end{array}$ & Not clear \\
\hline $\begin{array}{l}\text { Braunschweig } \\
\text { et al., } 2015 \\
\text { [67] }\end{array}$ & $\begin{array}{l}\text { Acute lung } \\
\text { injury patients } \\
\text { (medical and } \\
\text { surgical) }\end{array}$ & RCT & 78 & $\begin{array}{l}52.5 \text { years in the } \\
\text { intervention } \\
\text { group, } 58.6 \text { years } \\
\text { in the intervention } \\
\text { group }\end{array}$ & 51.2 & $\begin{array}{l}23.4 \text { in the } \\
\text { intervention group, } \\
27.7 \text { in the control } \\
\text { group }\end{array}$ & & $\begin{array}{l}\text { Intensive medical } \\
\text { nutrition: }>75 \% \text { of } \\
\text { estimated energy } \\
\text { and protein needs } \\
\text { by a multifaceted } \\
\text { approach ( } 1798 \pm \\
509 \mathrm{kcal} ; 25.4 \pm \\
6.6 \mathrm{kcal} / \mathrm{kg} / \text { day; } \\
84.7 \% \text { of energy } \\
\text { needs) Protein intake } \\
0.95 \mathrm{~g} / \mathrm{kg} / \text { day }\end{array}$ & $\begin{array}{l}\text { Standard nutrition } \\
\text { support care (1221 } \\
423 \mathrm{kcal} ; 16.6 \pm \\
5.6 \mathrm{kcal} / \mathrm{kg} / \text { day; } \\
55.4 \% \text { of energy } \\
\text { needs) Protein } \\
\text { intake } 0.68 \mathrm{~g} / \mathrm{kg} / \text { day }\end{array}$ & $\begin{array}{l}\text { Till hospital } \\
\text { discharge }\end{array}$ \\
\hline $\begin{array}{l}\text { Arabi et al., } \\
2015 \text { [68] }\end{array}$ & $\begin{array}{l}\text { Medical and } \\
\text { surgical }\end{array}$ & $\mathrm{RCT}$ & 894 & $\begin{array}{l}50.2 \text { years in the } \\
\text { intervention } \\
\text { group, } 50.9 \text { years } \\
\text { in the control } \\
\text { group }\end{array}$ & 64.2 & $\begin{array}{l}21.0 \text { in the } \\
\text { intervention and } \\
\text { control groups }\end{array}$ & $96.8 \%$ & $\begin{array}{l}\text { Permissive } \\
\text { underfeeding 40- } \\
60 \% \text { of caloric } \\
\text { requirements: (835 } \\
297 \mathrm{kcal} ; 46 \% \text { of } \\
\text { requirement) Protein } \\
\text { intake } 0.72 \mathrm{~g} / \mathrm{kg} / \text { day }\end{array}$ & $\begin{array}{l}\text { Standard feeding: } \\
70-100 \% \text { of caloric } \\
\text { requirements ( } 1299 \pm \\
467 \text { kcal; } 71 \% \text { of } \\
\text { requirement) Protein } \\
\text { intake } 0.73 \mathrm{~g} / \mathrm{kg} / \text { day }\end{array}$ & Up to 14 days \\
\hline $\begin{array}{l}\text { Doig et al., } \\
2015 \text { [69] }^{a^{\prime}}\end{array}$ & $\begin{array}{l}\text { Medical and } \\
\text { surgical }\end{array}$ & RCT & 331 & $\begin{array}{l}59 \text { years in the } \\
\text { intervention group, } \\
61 \text { years in the } \\
\text { control group }\end{array}$ & 58.6 & $\begin{array}{l}18 \text { in the intervention } \\
\text { and control groups }\end{array}$ & $91 \%$ & $\begin{array}{l}\text { Protocolized caloric } \\
\text { restriction } 20 \mathrm{kcal} / \mathrm{h} \\
\text { for } \geq 2 \text { days then } \\
\text { caloric intake adjusted } \\
\text { depending on serum } \\
\text { phosphate }\end{array}$ & $\begin{array}{l}\text { Standard care, mean } \\
\text { caloric intake at } \\
\text { enrolment } 68.5 \mathrm{kcal} / \mathrm{h}\end{array}$ & At least 4 days \\
\hline
\end{tabular}

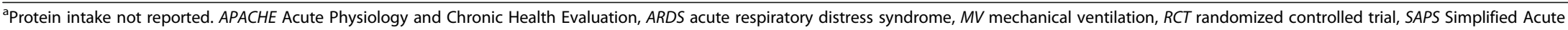
Physiology Score. SOFA Sequential Organ Failure Assessment 
Table 2 Quality of included randomized controlled trials using the Cochrane Collaboration tool for assessing risk of bias

\begin{tabular}{|c|c|c|c|c|c|c|c|c|c|}
\hline & Sequence generation & Concealment & Blinding & $\begin{array}{l}\text { Incomplete outcome } \\
\text { data }\end{array}$ & $\begin{array}{l}\text { Selective } \\
\text { outcome } \\
\text { reporting }\end{array}$ & $\begin{array}{l}\text { Other sources } \\
\text { of bias }\end{array}$ & Study center & $\begin{array}{l}\text { Percentage of patients } \\
\text { lost to follow up }\end{array}$ & $\begin{array}{l}\text { Source of study } \\
\text { funding }\end{array}$ \\
\hline $\begin{array}{l}\text { Montecalvo et al., } \\
1992 \text { [54] }\end{array}$ & Computer generated & Not described & No & No & No & No & $\begin{array}{l}\text { Multiple ICUs, } \\
\text { two } \\
\text { centers }\end{array}$ & 0 & Not reported \\
\hline $\begin{array}{l}\text { Kearns et al., } \\
2000[55]\end{array}$ & Computer generated & Sealed envelope & No & No & No & No & Single & 0 & $\begin{array}{l}\text { Ross Laboratories } \\
\text { and the California } \\
\text { Institute for } \\
\text { Medical Research } \\
\text { (partly) }\end{array}$ \\
\hline $\begin{array}{l}\text { Chen et al., } \\
2006 \text { [56] }\end{array}$ & Not described & Not described & No & No & No & No & $\begin{array}{l}\text { Two ICUs, } \\
\text { one center }\end{array}$ & 0 & $\begin{array}{l}\text { National Science } \\
\text { Council }\end{array}$ \\
\hline $\begin{array}{l}\text { Nguyen et al., } \\
2007 \text { [57] }\end{array}$ & Computer generated & Yes & Yes & No & No & No & Single & 0 & $\begin{array}{l}\text { National Health } \\
\text { and Medical } \\
\text { Research Council } \\
\text { (NHMRC) of } \\
\text { Australia (partly) }\end{array}$ \\
\hline $\begin{array}{l}\text { Desachy et al., } \\
2008 \text { [58] }\end{array}$ & Not described & Not described & No & No & No & No & Two ICUs & 0 & Not reported \\
\hline $\begin{array}{l}\text { Hsu et al., } \\
2009 \text { [59] }\end{array}$ & Computer generated & Yes & No & No & No & No & Single & 0 & $\begin{array}{l}\text { Kaohsiung } \\
\text { Veterans General } \\
\text { Hospital }\end{array}$ \\
\hline $\begin{array}{l}\text { White et al., } \\
2009 \text { [60] }\end{array}$ & Computer generated & $\begin{array}{l}\text { Yes (sealed opaque } \\
\text { envelope) }\end{array}$ & No & Yes & No & No & Single & 0 & Not reported \\
\hline $\begin{array}{l}\text { Montejo et al., } \\
2010 \text { [61] }\end{array}$ & Not described & Not described & No & No & No & No & Single & 0 & Not reported \\
\hline $\begin{array}{l}\text { Acosta-Escribano } \\
\text { et al., } 2010 \text { [62] }\end{array}$ & Central randomization & Yes & No & Yes & No & No & Multicenter & 0 & $\begin{array}{l}\text { Novartis } \\
\text { Consumer } \\
\text { Health (Spain) }\end{array}$ \\
\hline $\begin{array}{l}\text { Arabi et al., } \\
2011 \text { [14] }\end{array}$ & Computer generated & Yes & No & No & No & No & Single & 0 & $\begin{array}{l}\text { King Abdulaziz } \\
\text { City for Science } \\
\text { and Technology }\end{array}$ \\
\hline $\begin{array}{l}\text { Singer et al., } \\
2011 \text { [63] }\end{array}$ & Computer generated & Yes & No & No & No & No & Single & 0 & Not reported \\
\hline $\begin{array}{l}\text { Rice et al., } \\
2011 \text { [15] }\end{array}$ & Not described & $\begin{array}{l}\text { Yes (sealed opaque } \\
\text { envelope) }\end{array}$ & No & No & No & No & $\begin{array}{l}\text { Two ICUs, } \\
\text { single center }\end{array}$ & 0 & $\begin{array}{l}\text { National } \\
\text { Institutes of } \\
\text { Health (partly) }\end{array}$ \\
\hline $\begin{array}{l}\text { Rice et al., } \\
2012 \text { [16] }\end{array}$ & Web-based system & Sealed envelope & No & No & No & No & Multicenter & 0 & $\begin{array}{l}\text { National Heart, } \\
\text { Lung, and Blood } \\
\text { Institute }\end{array}$ \\
\hline $\begin{array}{l}\text { Huang et al., } \\
2012 \text { [64] }\end{array}$ & $\begin{array}{l}\text { Software-generated } \\
\text { randomization }\end{array}$ & Not described & No & $\begin{array}{l}\text { Yes Hospital mortality } \\
\text { data were missing } \\
\text { for some patients }\end{array}$ & No & No & Single & $\begin{array}{l}\text { Hospital mortality data } \\
\text { missing for } 4 / 101 \\
\text { patients (4\%) }\end{array}$ & $\begin{array}{l}\text { Kaohsiung } \\
\text { Veterans General } \\
\text { Hospital }\end{array}$ \\
\hline
\end{tabular}


Table 2 Quality of included randomized controlled trials using the Cochrane Collaboration tool for assessing risk of bias (Continued)

\begin{tabular}{|c|c|c|c|c|c|c|c|c|c|}
\hline $\begin{array}{l}\text { Reignier et al., } \\
2013 \text { [65] }\end{array}$ & $\begin{array}{l}\text { Computer-generated, } \\
\text { interactive, web- } \\
\text { response system }\end{array}$ & Yes & No & No & No & No & Multicenter & 0 & $\begin{array}{l}\text { The Centre } \\
\text { Hospitalier } \\
\text { Departemental } \\
\text { de la Vendee }\end{array}$ \\
\hline $\begin{array}{l}\text { Rugeles et al., } \\
2013 \text { [23] }\end{array}$ & $\begin{array}{l}\text { Computer-generated } \\
\text { random allocations }\end{array}$ & $\begin{array}{l}\text { No } \\
\text { Sealed envelopes } \\
\text { were used but one } \\
\text { investigator knew } \\
\text { patient allocation }\end{array}$ & $\begin{array}{l}\text { No } \\
\text { One } \\
\text { investigator } \\
\text { knew patient } \\
\text { allocation. }\end{array}$ & $\begin{array}{l}\text { Yes } \\
\text { Mortality was } \\
\text { reported as one } \\
\text { of the secondary } \\
\text { endpoints but was } \\
\text { not reported }\end{array}$ & Yes & $\begin{array}{l}\text { Yes } \\
\text { Many patients } \\
\text { were excluded from } \\
\text { analysis with } \\
\text { uncertainty if } \\
\text { exclusion criteria } \\
\text { were determined a } \\
\text { priori. }\end{array}$ & Single & 0 & Lafrancol S.A \\
\hline $\begin{array}{l}\text { Peake et al., } \\
2014 \text { [66] }\end{array}$ & Web-based system & $\begin{array}{l}\text { Centralized, web- } \\
\text { based randomization } \\
\text { schedule }\end{array}$ & Yes & No & No & No & Multicenter & $\begin{array}{l}\text { One patient in the } \\
\text { intervention group } \\
\text { was withdrawn and } \\
\text { one patient in the } \\
\text { control group was } \\
\text { lost to follow up by } \\
\text { day } 90 \text {. }\end{array}$ & $\begin{array}{l}\text { The Royal } \\
\text { Adelaide Hospital } \\
\text { and the } \\
\text { Australian, New } \\
\text { Zealand College } \\
\text { of Anaesthetists } \\
\text { and Fresenius } \\
\text { Kabi }\end{array}$ \\
\hline $\begin{array}{l}\text { Charles et al., } \\
2014 \text { [22] }\end{array}$ & $\begin{array}{l}\text { Random number } \\
\text { sequence }\end{array}$ & $\begin{array}{l}\text { Opaque security } \\
\text { envelopes }\end{array}$ & No & No & No & $\begin{array}{l}\text { Yes } \\
\text { The trial was } \\
\text { stopped before } \\
\text { achieving the target } \\
\text { sample size because } \\
\text { of slow enrolment }\end{array}$ & Single & 0 & The NIH \\
\hline $\begin{array}{l}\text { Braunschweig et al., } \\
2015 \text { [67] }\end{array}$ & $\begin{array}{l}\text { Computer-generated } \\
\text { random allocations }\end{array}$ & Sealed envelopes & No & No & No & $\begin{array}{l}\text { Yes } \\
\text { The trial was } \\
\text { stopped before } \\
\text { achieving the target } \\
\text { sample size because } \\
\text { of higher mortality, } \\
\text { a secondary } \\
\text { outcome, in the } \\
\text { intervention group }\end{array}$ & Single & 0 & The NIH/NHLBI \\
\hline $\begin{array}{l}\text { Arabi et al., } \\
2015 \text { [68] }\end{array}$ & $\begin{array}{l}\text { Computer-generated } \\
\text { random allocations } \\
\text { (blocks of variable size }\end{array}$ & $\begin{array}{l}\text { Opaque sealed } \\
\text { envelopes }\end{array}$ & No & No & No & No & Multicenter & $\begin{array}{l}9 \text { patients lost to } \\
\text { follow up, } 3 \text { in the } \\
\text { intervention group } \\
\text { and } 3 \text { in the control } \\
\text { group }\end{array}$ & $\begin{array}{l}\text { King Abdullah } \\
\text { International } \\
\text { Medical Research } \\
\text { Center }\end{array}$ \\
\hline $\begin{array}{l}\text { Doig et al., } \\
2015 \text { [69] }\end{array}$ & $\begin{array}{l}\text { Computer-generated } \\
\text { random allocations } \\
\text { (blocks of variable size) }\end{array}$ & $\begin{array}{l}\text { Secure central } \\
\text { randomization } \\
\text { web server }\end{array}$ & No & No & No & No & Multicenter & $\begin{array}{l}4 \text { patients lost to } \\
\text { follow up ( } 90 \text {-day } \\
\text { interview), } 2 \text { in each } \\
\text { group. }\end{array}$ & $\begin{array}{l}\text { National Health } \\
\text { and Medical } \\
\text { Research Council } \\
\text { of Australia }\end{array}$ \\
\hline
\end{tabular}




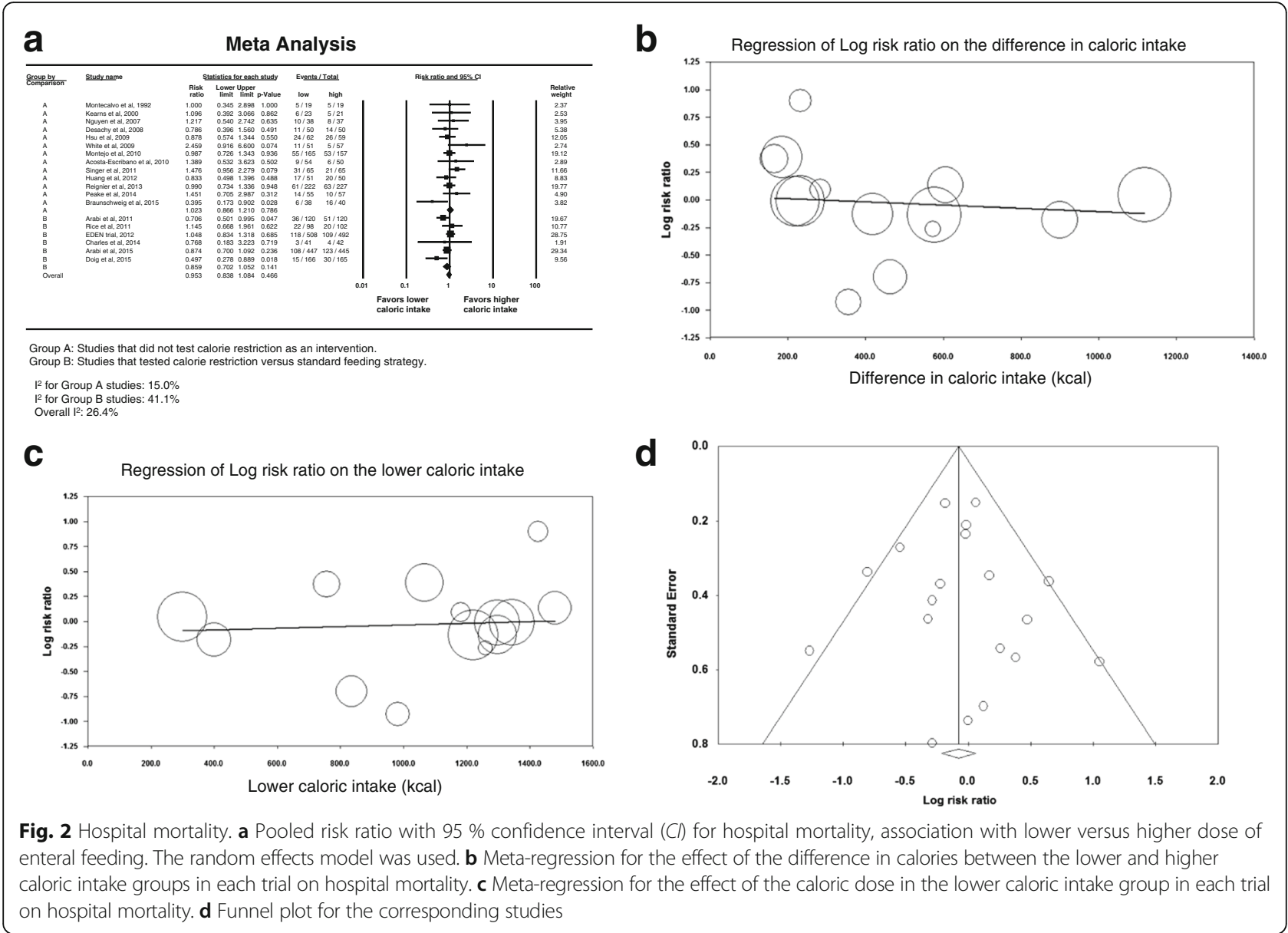

significant heterogeneity among the studies $\left(I^{2}=0 \%\right)$ and no evidence of publication bias (Egger's test $p=0.40$ ).

\section{Effect on infections}

Twelve studies reported data on more than one infection [14-16, 22, 54, 55, 62, 63, 65, 67-69]. Figure 4 describes the corresponding forest plot. The overall RR was 0.972 (95\% CI, 0.878-1.077) with no significant difference between caloric restriction and non-caloric-restriction trials (interaction test $p=0.91$ ). The overall heterogeneity was low $\left(I^{2}=25.7 \%\right)$.

Eleven studies collected data on bloodstream infections $[14,16,22,54,55,59,62,63,65,68,69]$ and showed lower risk of such infections in the lower versus higher caloric intake groups (RR 0.718; 95 \% CI 0.5190.994; Fig. 5a). The interaction test between caloric restriction and non-caloric-restriction trials was not significant $(p=0.48)$. Meta-regression showed no association between bloodstream infection risk and the amount of calorie intake in the difference in caloric intake between the intervention groups in each trial (slope $=0.0001 ; p=0.75$ ) and the lower calorie group in each trial (slope $=-0.0002$; $p=0.30$ ) (Fig. $5 \mathrm{~b}$ and c). There was low heterogeneity among the studies $\left(I^{2}=26.7 \%\right)$. The funnel plot (Fig. $5 \mathrm{~d}$ ) showed possible publication bias (Egger's test $p=0.04$ ).

Fifteen studies reported data on development of pneumonia [14-16, 22, 54-56, 59-63, 65, 68, 69]. Figure 6 presents the related forest plot. The overall RR was 0.920 (95\% CI 0.784-1.080) with no significant difference between caloric restriction and non-caloric-restriction trials (interaction test $p=0.88$ ). There was substantial heterogeneity among the studies $\left(I^{2}=53.8 \%\right)$.

\section{Effect on incident renal replacement therapy}

Five studies reported data on incident renal replacement therapy $[14,63,65,68,69]$. The overall RR was 0.711 (95\% CI 0.545-0.928) (Fig. 7a) with no significant difference between caloric restriction and non-caloric-restriction trials (test of interaction $p=0.33$ ). The meta-regression (Fig. 7b and c) showed significant association between incident renal replacement therapy and the difference in caloric intake between the lower and higher calorie groups in each study (slope $=-0.0009 ; p=0.02$ ) and the lower 


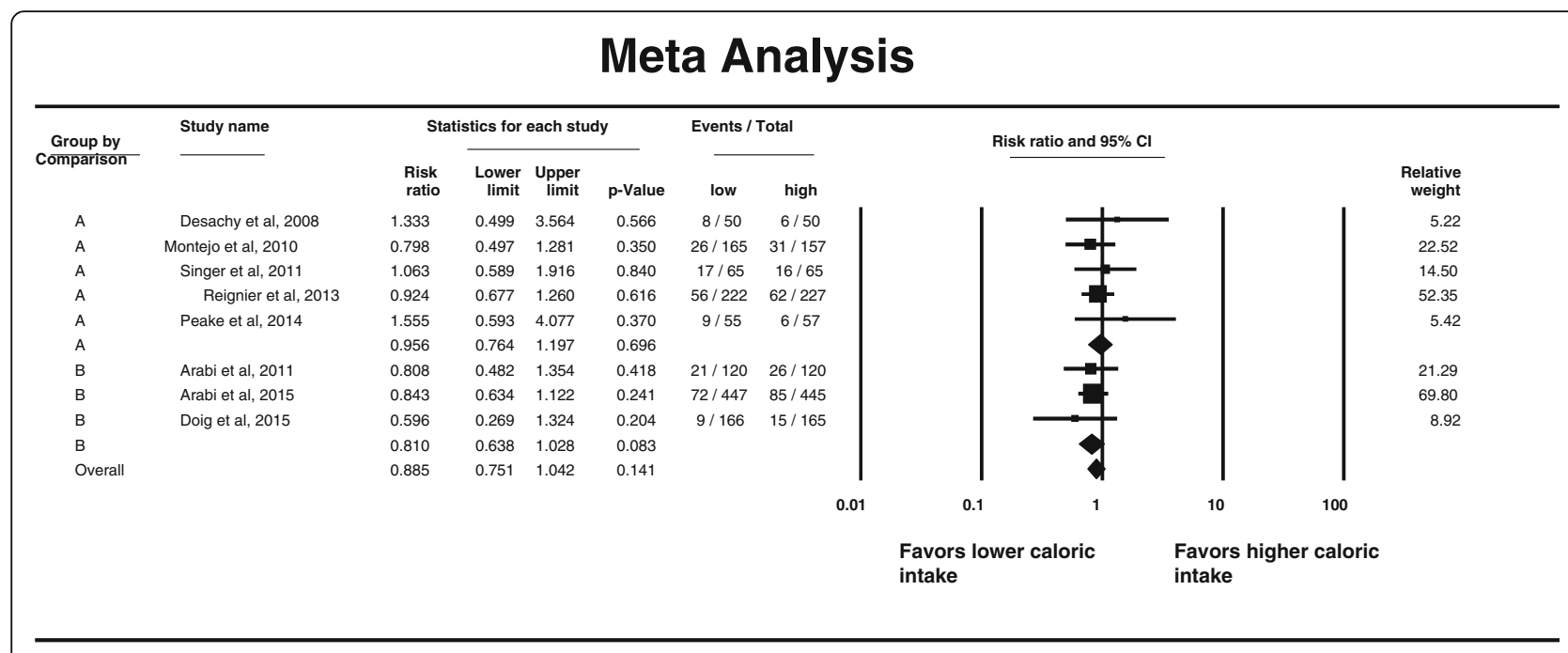

Group A: Studies that did not test calorie restriction as an intervention.

Group B: Studies that tested calorie restriction versus standard feeding strategy.

$I^{2}$ for Group A studies: 0\%

${ }^{2}$ for Group B studies: 0\%

Overall 12: 0\%

Fig. 3 Pooled risk ratio with $95 \%$ confidence interval $(C l)$ for intensive care unit mortality associated with lower versus higher dose of enteral feeding. The random effects model was used

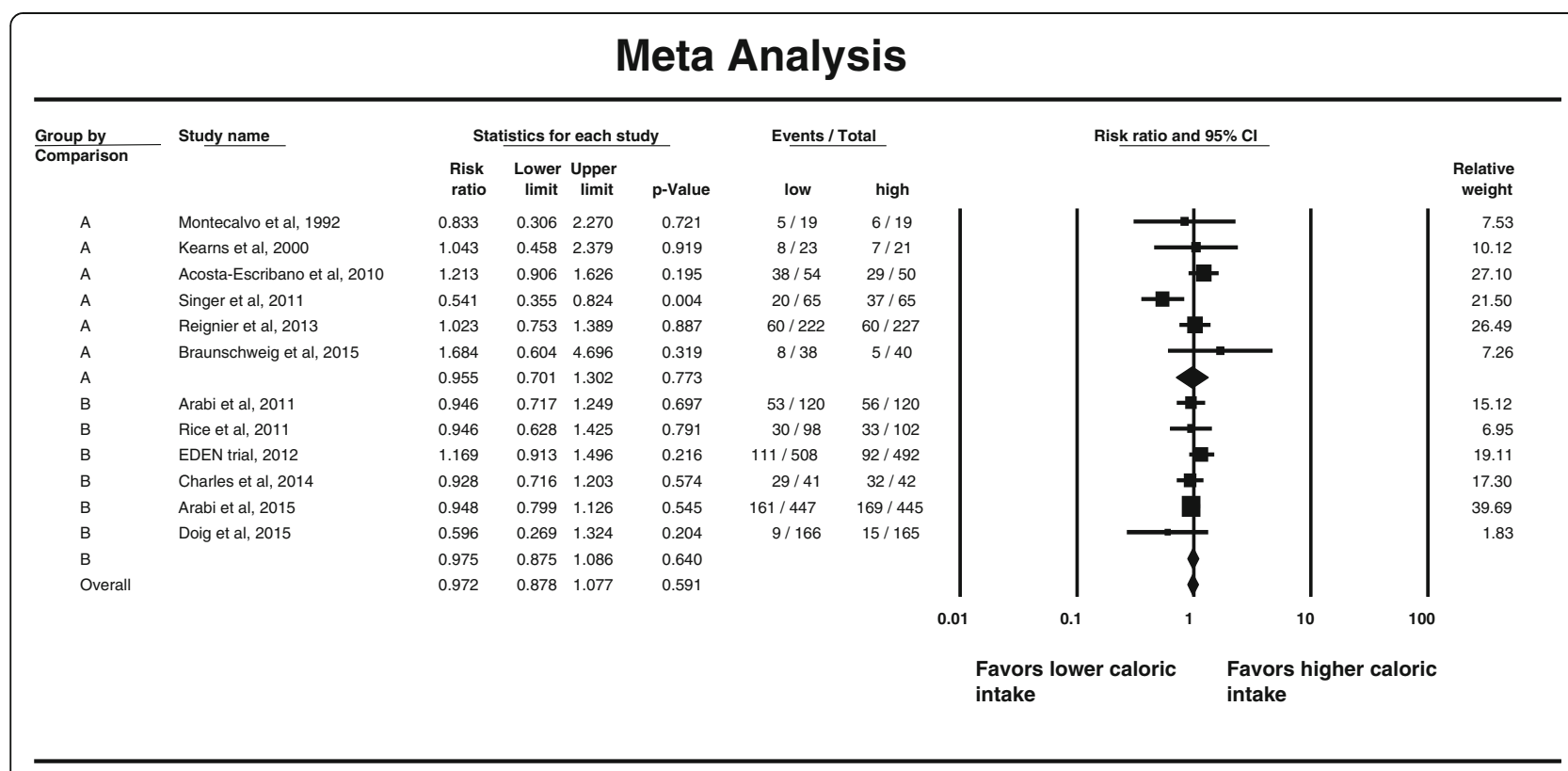

Group A: Studies that did not test calorie restriction as an intervention.

Group B: Studies that tested calorie restriction versus standard feeding strategy.

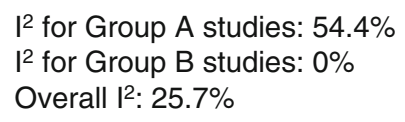

Fig. 4 Pooled risk ratio with $95 \%$ confidence interval $(C l)$ for total infections associated with lower versus higher dose of enteral feeding. The random effects model was used 


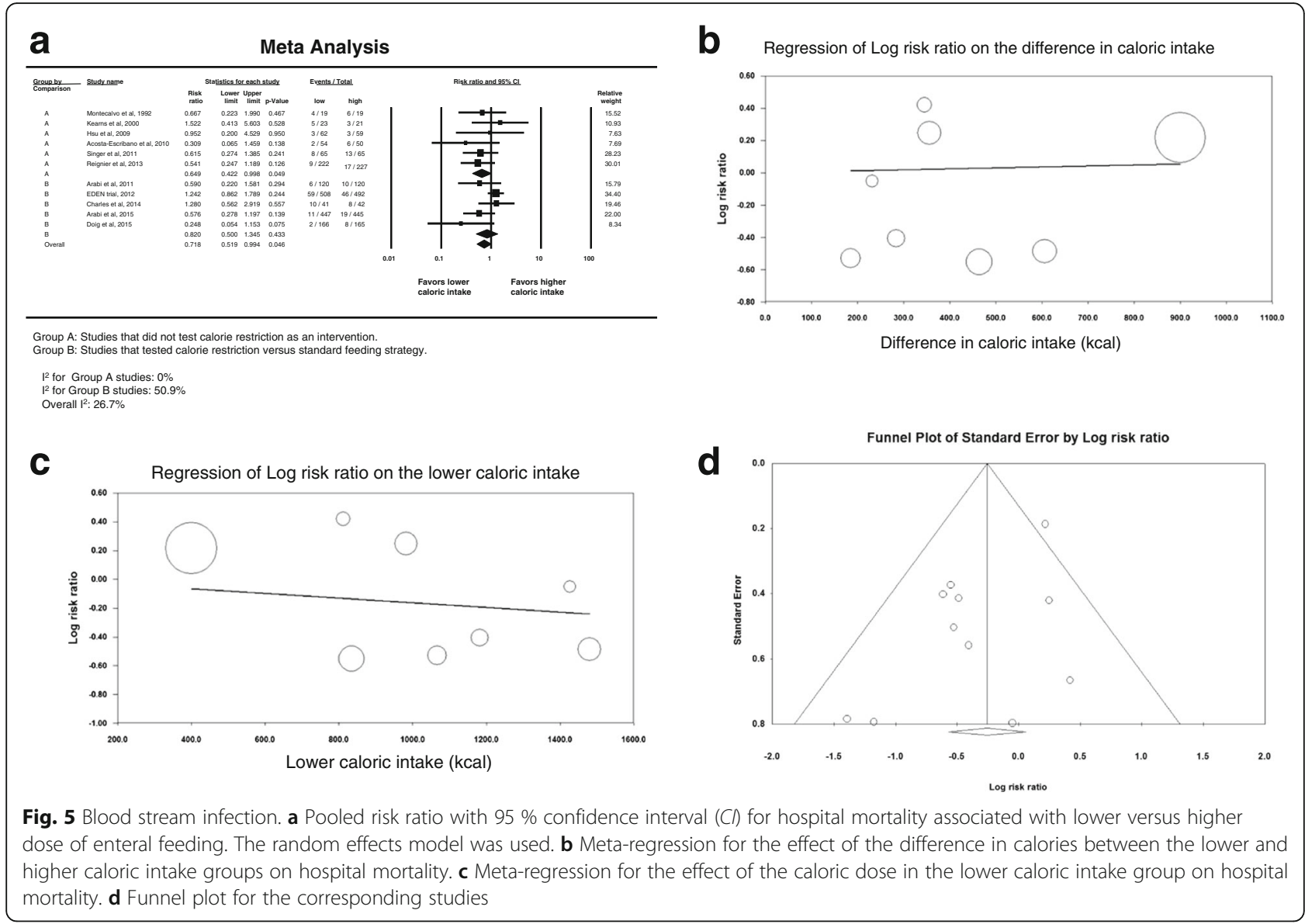

caloric dose in each study (slope $=-0.0004 ; p=0.02$ ). There was low heterogeneity $\left(I^{2}=0 \%\right)$ with the funnel plot shown in Fig. 7d (Egger's test $p=0.17$ ).

\section{Effect on length of stay}

Eleven studies provided data (means with standard deviations) on hospital LOS [14, 22, 55, 57-60, 62, 67-69]. The pooled analysis showed no difference in hospital stay $(\mathrm{WMD}=+1.11$ days; $95 \%$ CI $-1.09-3.30$ days) (Fig. 8). However, the association of lower versus higher caloric intake and hospital LOS was different between caloric restriction and non-caloric-restriction trials (interaction test $p=0.005$ ). In the caloric restriction trials, the hospital LOS was longer with lower compared with higher caloric intake (WMD $=+4.09$ days; $95 \%$ CI 1.08-7.11 days), whereas it was not different between lower and higher dose caloric intake in the non-caloric restriction trials (Fig. 8). There was substantial heterogeneity among the studies $\left(I^{2}=76.3 \%\right)$. However, there was no evidence of publication bias (Egger's test $p=0.11$ ). Meta-regression analysis showed that the hospital LOS was not influenced by the difference in caloric intake between the lower and higher calorie groups in each trial or by the lower calorie dose in each trial.
Fourteen studies provided data on ICU LOS [14, 22, $23,54,55,58,59,61-64,67-69]$ with no significant difference between the lower and higher caloric intake groups (WMD $=-0.13$ days (95\% CI $-1.45-1.19$ days) (Fig. 9). The association between lower compared with higher caloric intake and ICU LOS was not different between caloric restriction and non-caloric-restriction trials (test of interaction $p=0.39$ ). There was a substantial heterogeneity among the studies $\left(I^{2}=78.0 \%\right)$. Egger's test $p$ was 0.07 .

Eight studies had data on duration of mechanical ventilation $[14,23,54,59,62,63,68,69]$. The meta-analysis found no difference (Fig. 10) with WMD $=-1.12$ days (95 \% CI -2.67- 0.44 days). There was substantial heterogeneity among the studies $\left(I^{2}=69.7 \%\right)$. The association between lower compared with higher caloric intake and mechanical ventilation duration was not different between caloric restriction and non-caloric-restriction trials (test of interaction $p=0.89$ ). The Egger's test $p$ value was 0.09 , suggesting insignificant publication bias.

\section{Discussion}

In this systematic review and meta-analysis of RCTs of critically ill patients in which there was a significant 


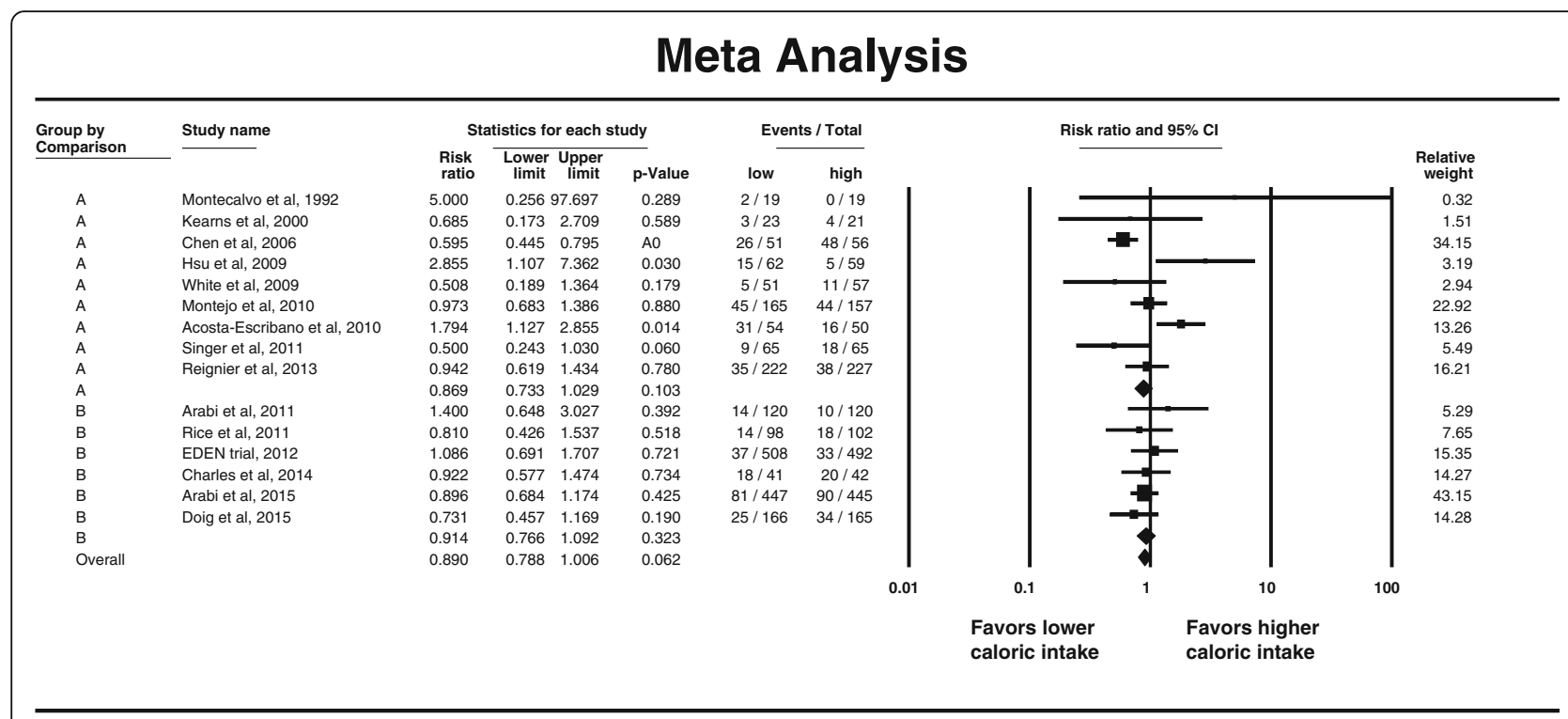

Group A: Studies that did not test calorie restriction as an intervention.

Group B: Studies that tested calorie restriction versus standard feeding strategy.

$\mathrm{I}^{2}$ for Group Astudies:70.7\%

$\mathrm{I}^{2}$ for Group B studies: 0\%

Overall ${ }^{2}: 53.8 \%$

Fig. 6 Pooled risk ratio with $95 \%$ confidence interval $(C l)$ for pneumonia associated with lower versus higher dose of enteral feeding. The random effects model was used

difference in the caloric intake via $\mathrm{EN}$, we found that lower versus higher dose of caloric intake was not associated with mortality. However, patients in the lower caloric intake group had lower risk of blood stream infections and incident renal replacement therapy. The associations between lower compared with higher caloric intake and all studied outcomes were not different between caloric restriction and non-caloric restriction trials, except for the hospital LOS. Our certainty in the metaanalytic estimates is reduced due to the moderate risk of bias.

The optimal EN dose during critical illness is unknown. Malnourishment is generally associated with poor outcomes leading to the notion that replacement of full energy needs is intuitively needed. However, in critically ill patients, multiple metabolic changes may occur and differ from one patient to another. The metabolic rate is highly variable, being increased in about $50 \%$ of patients but low in others [70]. Glycolysis is increased as the energy source is altered from predominantly fat to glucose oxidation [71]. Proteolysis is accelerated especially as prolonged starvation is associated with dysregulated metabolism, such that major stimulation of both ketogenesis and concomitant gluconeogenesis do not occur [72]. The normal suppression of lipolysis and proteolysis by the exogenous supply of fat or carbohydrates is blunted [71]. Additionally, excess caloric provision might be detrimental, particularly to the mitochondria by increasing oxygen radical production [73], and full EN may lead to gastrointestinal intolerance, which may increase infection risk. On the other hand, caloric restriction may enhance autophagy, which might be protective $[74,75]$. These changes have led to the premise that lower caloric intake may have a protective effect.

The available studies on this topic produced mixed results. In this meta-analysis of RCTs in which critically ill patients received significantly different caloric intake, we found no difference in the primary outcome of hospital mortality. The results were similar in caloric restriction and non-caloric restriction trials and when studies were stratified based on age, severity of illness, amount of calories in the lower calorie group and the difference in calories between the intervention groups. Moreover, there was no clear dose-effect relationship as observed in the metaregression. Marik and Hooper have recently published a systematic review and meta-analysis of six trials that assessed the outcomes of ICU patients randomized to either normocaloric or hypocaloric feeding and also found no difference in mortality and infectious complications [76]. However, our study differed in its inclusion and exclusion criteria and thus included 21 trials, which evaluated various interventions that resulted in different caloric intake. We also analyzed multiple subgroups and performed meta-regression. 


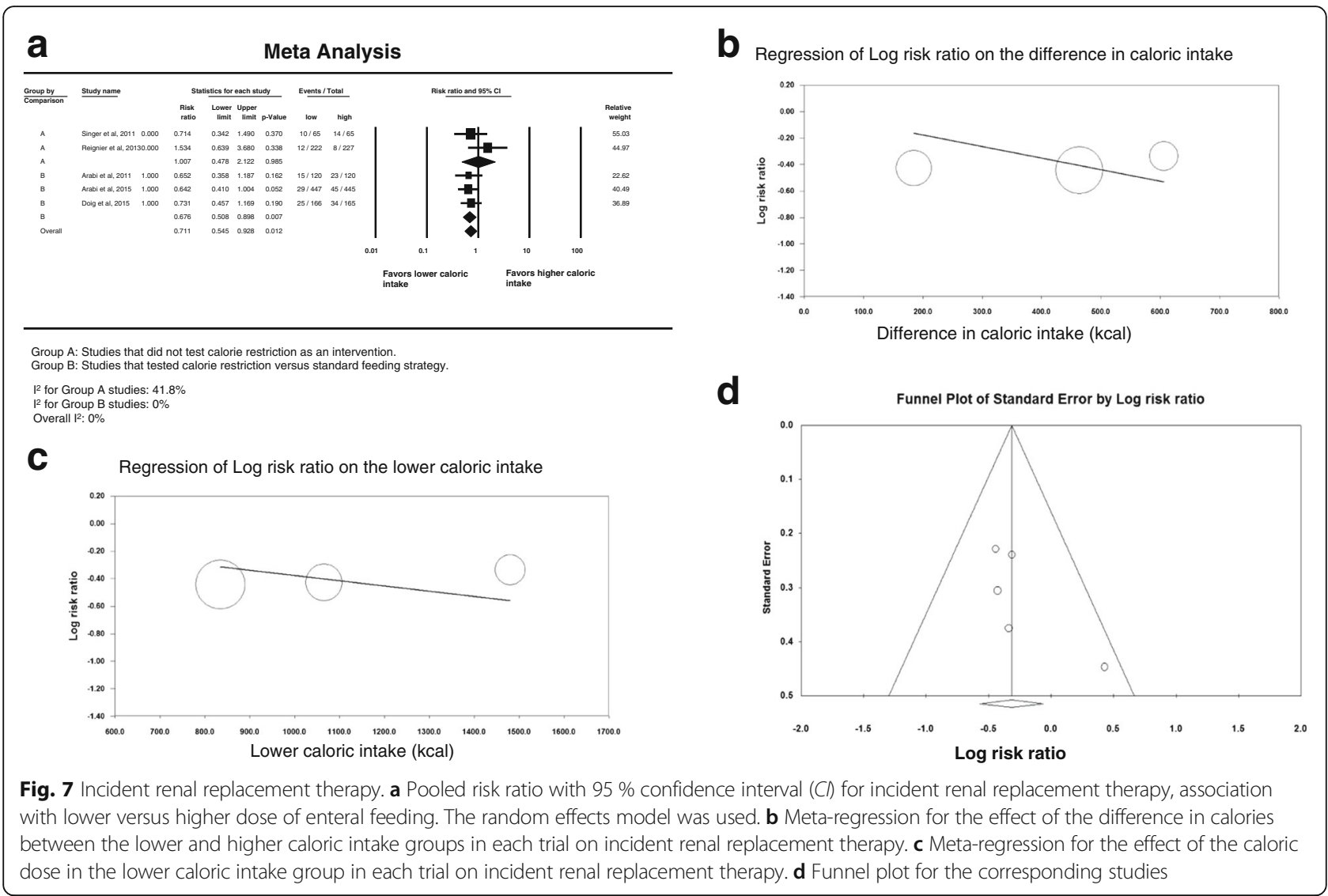

We note that in one of the included trials, the TICACOS trial [63], the difference in energy intake was largely provoked by greater administration of $\mathrm{PN}$ rather than EN. However, we included the study because it met our a priori inclusion criterion; the mean enterally delivered energy in the study group was $1515 \pm 756 \mathrm{kcal} /$ day compared with $1316 \pm 456 \mathrm{kcal} /$ day in the control group $(p=0.09)$, which is more than $10 \%$ [63]. We also note that in the included INTACT trial [67], the number of days between hospital admission and enrollment were $8.8 \pm 8.7$ and 6.4 \pm 6.6 days in the intensive medical and standard nutrition groups, respectively (Table 1 ) and the caloric target was $30 \mathrm{kcal} / \mathrm{kg}$ in the intensive medical group. These issues raised concerns about the occurrence of refeeding syndrome, which was not studied in the trial, and of overfeeding in the intensive medical group which may have led to early ICU deaths [67, 77-79].

The relationship between nutrition and infection risk has been evaluated in different settings. A meta-analysis found that EN versus PN was associated with a significant decrease in infectious complications in critically ill patients (relative risk 0.64, $95 \%$ CI $0.47-0.87 ; p=0.004$ ) [80]. Another meta-analysis found that EN within 24 hours after elective gastrointestinal surgery compared with nil-by-mouth management reduced any infection risk (relative risk 0.72, $95 \%$ CI 0.54-0.98; $p=0.04$ ) [81]. However, the impact of EN dose in critically ill patients is not well-studied. In the current study, we have observed that lower caloric intake was associated with lower nosocomial blood stream infection risk. However, there was no effect on total infections or pneumonia. This could be related to the heterogeneity of studies and publication bias.

Caloric restriction has been shown to be reno-protective in animal models of acute kidney injury [82-84]. However, a secondary analysis in the Randomized Evaluation of Normal vs. Augmented Level of Replacement Therapy trial found that the increased caloric intake was associated with lower 90-day mortality on multivariable logistic regression analysis (odds ratio per $100 \mathrm{kcal}$ increment 0.95 , $95 \%$ CI $0.91-1.00 ; p=0.06)$ on multivariable logistic regression analysis [85]. This was more pronounced after excluding patients who died within 96 hours of ICU admission [85]. However, the mean caloric intake during treatment was low (approximately $11 \pm$ $9 \mathrm{kcal} / \mathrm{kg} /$ day). Our meta-analysis identified lower incident renal replacement therapy rates in the lower calorie group, but this finding is limited by the small number of studies $(n=5)$ that reported on this outcome. Other possible mechanisms for the difference in renal replacement therapy, which may include differences in protein 


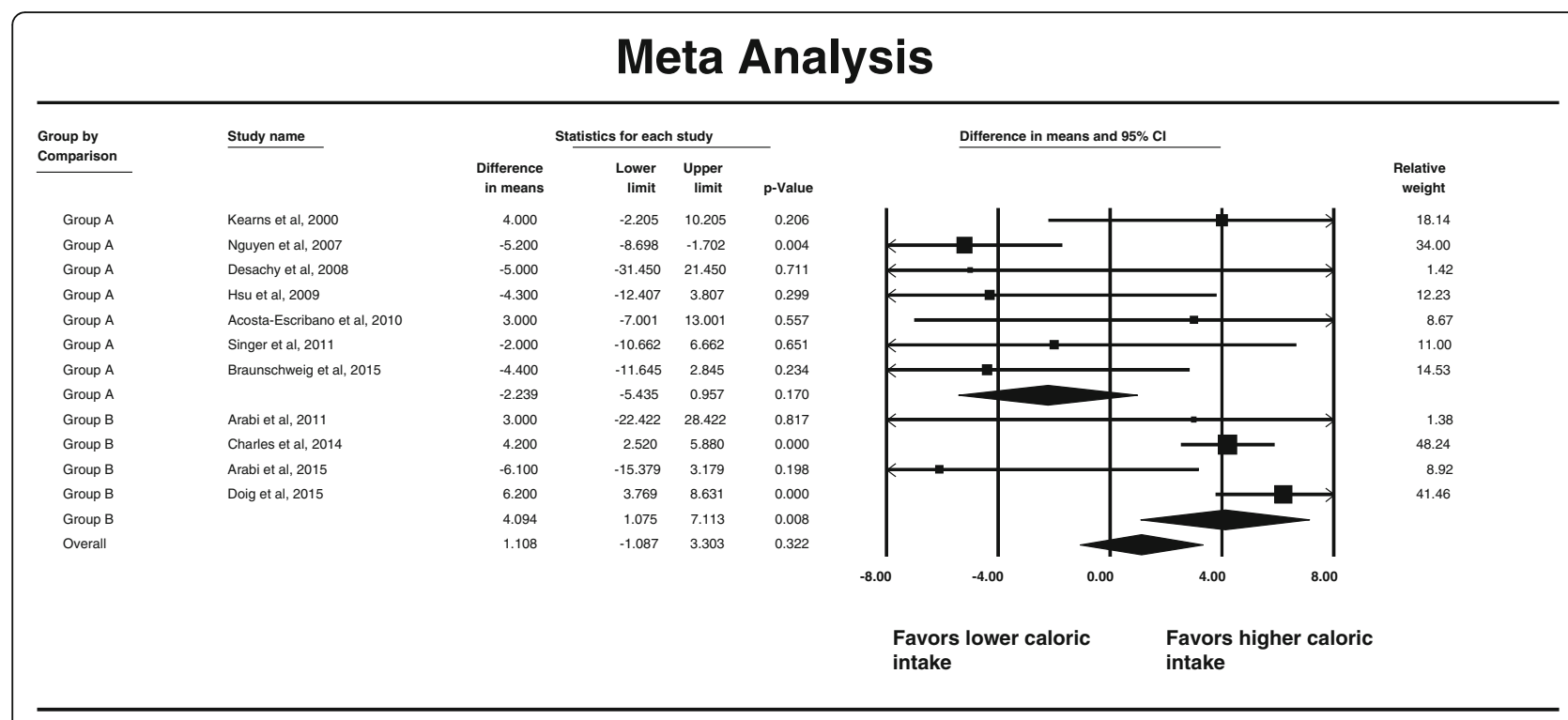

Group A: Studies that did not test calorie restriction as an intervention.

Group B: Studies that tested calorie restriction versus standard feeding strategy.

$I^{2}$ for Group A studies: $25.8 \%$

I $^{2}$ for Group B studies: $57.1 \%$

Overall $\mathrm{I}^{2}: 76.3 \%$

Fig. 8 Pooled risk ratio with $95 \%$ confidence interval $(C /)$ for hospital length of stay associated with lower versus higher dose of enteral feeding. The random effects model was used

intake or fluid balance between the two groups, could not be examined in our meta-analysis. Further studies are needed to clarify this association.

Multiple observational and interventional studies have identified variable associations between EN dose and ICU and hospital LOS and mechanical ventilation duration [9, 12, 86-88]. Our meta-analysis did not identify differences in mechanical ventilation duration or ICU LOS. Lower caloric intake was associated with longer hospital LOS in the subgroup of studies that compared caloric restriction with standard feeding.

This study needs to be interpreted in the light of its strengths and limitations. Potential confounders were controlled for because of the randomized design of the included trials. For example, age and severity of illness were similar in the two arms in the included trials (Table 1). A further strength is that rather than being selective and possibly biased in selecting trials, we included all studies in which a difference in enteral intake was provoked by the intervention. As such we included not only studies on caloric restriction but also studies evaluating other interventions that affect caloric intake, such as gastric residual volume management or small bowel feeding. Such relatively wide inclusion criteria allowed the inclusion of more trials. However, this strength may have provoked a possible weakness. Studies were pooled despite different design, some of them not even focused on improving outcome by reaching feeding goals or by nutrient restriction. We have addressed this limitation by our subgroup analyses and by the meta-regression. Our study focused on EN and did not include studies that had PN as the primary intervention. Hence, the study by Heidegger et al., in which ICU patients who received $<60 \%$ of their calorimetry-calculated caloric target from EN were randomized to either EN or EN plus supplemental PN [89], was not included. Although several studies showed similar outcomes with the two routes $[80,90]$, we chose not to include PN trials as this may further contribute to the heterogeneity. In addition, permissive underfeeding in $\mathrm{PN}$ has been studied in earlier systematic reviews $[8,91]$.

Another limitation is related to the quality of the included studies, most of which had one or more form of bias. Furthermore, the caloric intake in all included studies in the higher feeding group was generally less than the estimated caloric requirement. This is a reflection of the difficulty in achieving full targets in cohorts of ICU patients (although it certainly is achievable in many individual patients). However, this resembles what has been shown in many observational studies and in real-world practice. Given that the full enteral intake was reached in a few studies only, the benefit of this strategy remains unclear. While some studies calculated the calories from sources other than EN and PN, such as intravenous 


\section{Meta Analysis}

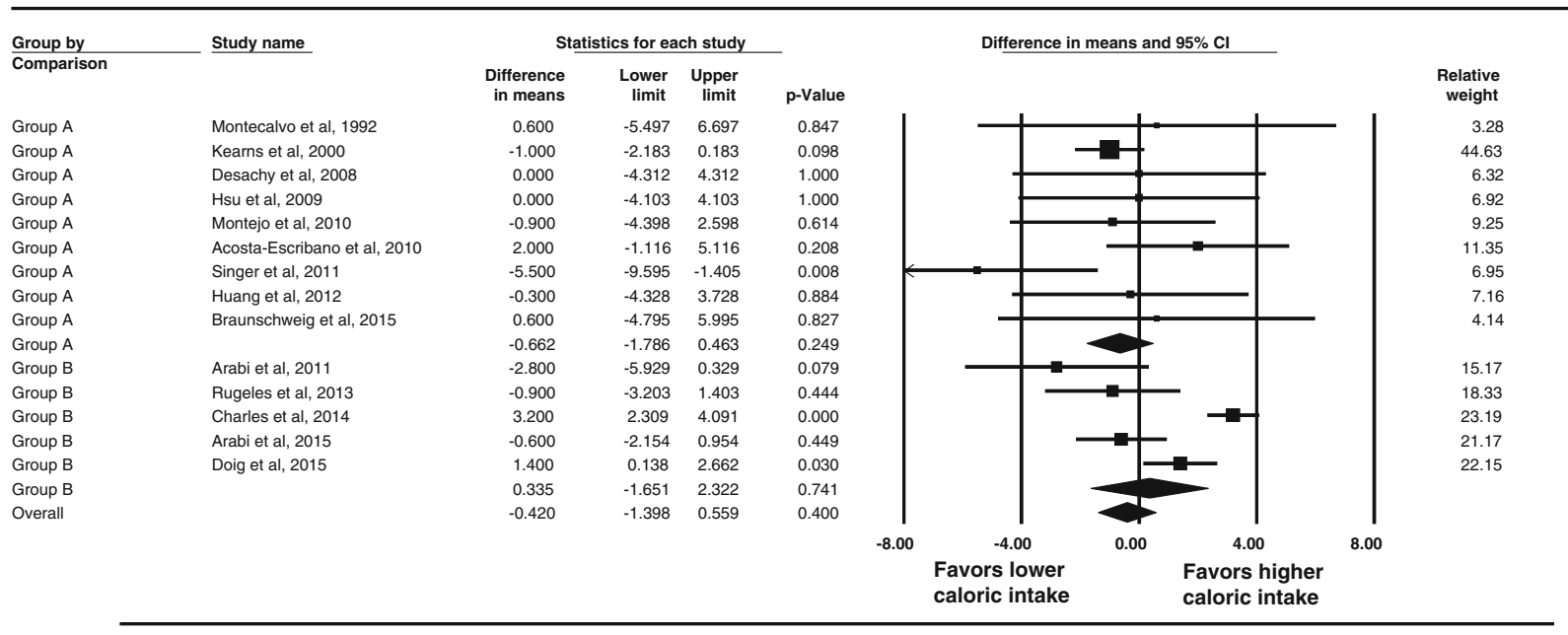

Group A: Studies that did not test calorie restriction as an intervention.

Group B: Studies that tested calorie restriction versus standard feeding strategy.

$I^{2}$ for Group A studies: $11.7 \%$

I2 for Group B studies: $87.6 \%$

Overall I2: $78.0 \%$

Fig. 9 Pooled risk ratio with $95 \%$ confidence interval $(C l)$ for intensive care unit length of stay associated with lower versus higher dose of enteral feeding. The random effects model was used

\section{Meta Analysis}

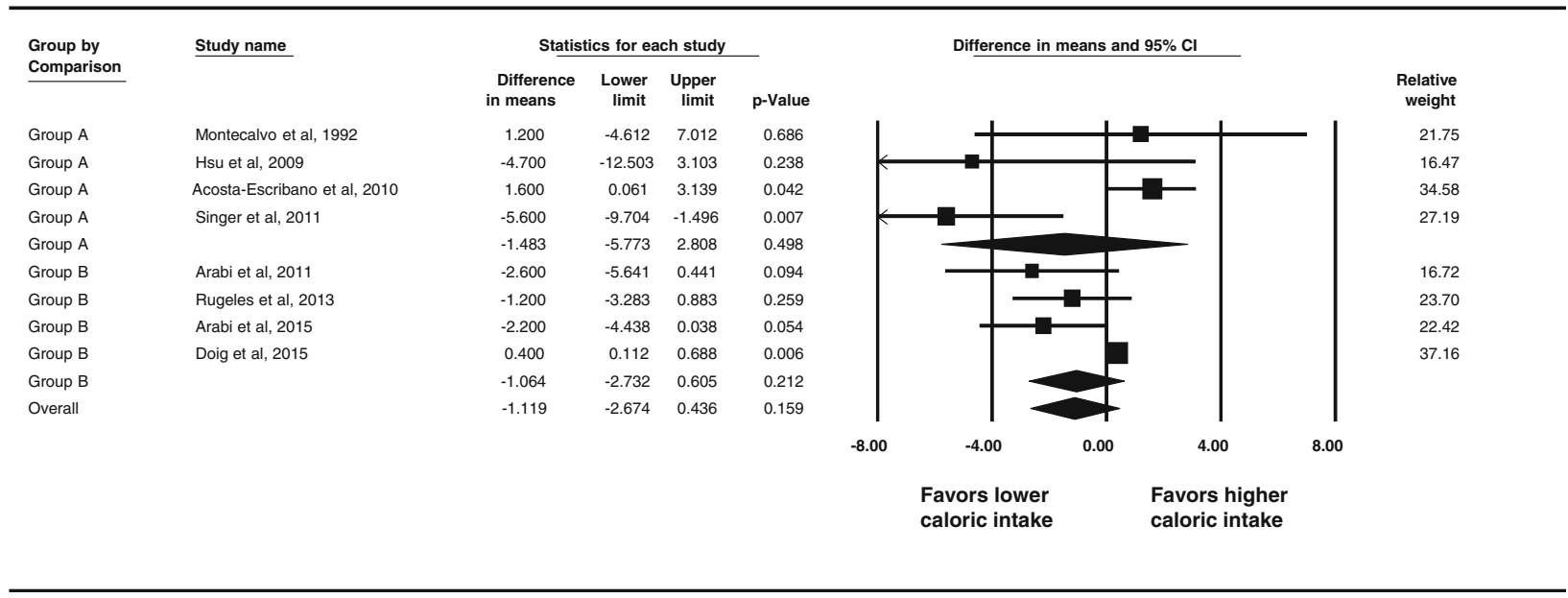

Group A: Studies that did not test calorie restriction as an intervention.

Group B: Studies that tested calorie restriction versus standard feeding strategy.

$I^{2}$ for Group A studies: $75.4 \%$

$\mathrm{I}^{2}$ for Group B studies: $72.1 \%$

Overall $\mathrm{I}^{2}: 69.7 \%$

Fig. 10 Pooled risk ratio with $95 \%$ confidence interval $(C l)$ for the duration of mechanical ventilation associated with lower versus higher dose of enteral feeding. The random effects model was used 
dextrose (including medications) and propofol $[14,55,63$, 66-68], this was not a consistent approach among all trials. Most included studies have used predictive equations rather than indirect calorimetry to estimate caloric intake. However, this is consistent with usual practice in most ICUs, given the limited evidence supporting either approach. Energy requirements were estimated using different predictive formulae in all but one study, which used indirect calorimetry [63]. This method has been shown to poorly correlate with indirect calorimetry [92], which is considered to be the standard. Based on recent evidence, calorimetry-guided nutrition might be superior to predictive formula-based feeding in improving patient-centered outcomes [63, 89].

The caloric dose and target varied among the included studies and what was lower caloric intake in one study was high in another. However, the meta-regression analysis suggested that the differences in calories between groups and the amount of calories in the lower caloric intake group did not influence hospital mortality. Moreover, not all studies reported on all the outcomes, thus reducing the sample size used and the ability to find small but potentially clinically significant effects. Given the reported data in the individual studies, further analysis of the impact of protein intake could not be performed. Our systemic review highlights the limited data on this important issue and calls for further studies on the impact of protein intake on the outcomes of critically ill patients. Our meta-analysis was not an individual-patient meta-analysis and did not include economic or long-term endpoints.

\section{Conclusions}

Lower versus higher dose of caloric intake in adult critically ill patients was not associated with differences in mortality, risk of pneumonia or mechanical ventilation duration. Lower caloric intake was associated with lower risk of blood stream infections and incident renal replacement. The great heterogeneity in the design, feeding route and timing and caloric dose among the included trials could limit the interpretation of the results of this systematic review and meta-analysis. Further studies are needed to further evaluate the association between caloric intake and renal replacement therapy in critically ill patients.

\section{Additional file}

Additional file 1: Search strategy. (DOC $56 \mathrm{~kb}$ )

\section{Abbreviations}

APACHE: Acute Physiology and Chronic Health Evaluation; Cl: Confidence interval; EN: Enteral nutrition; ICU: Intensive care unit; LOS: Length of stay; PN: Parenteral nutrition; PRISMA: Preferred Reporting Items for Systematic Reviews and Meta-analyses; RR: Risk ratio; RCT: Randomized controll ed trial; WMD: Weighted mean difference

\section{Acknowledgements}

Faisal Wali Ahmed, MD, Staff Physician, Intensive Care Department, King Abdulaziz Medical City, Riyadh, Saudi Arabia. Email: faisalwali786@gmail.com

\section{Funding}

None.

Availability of data and materials

Available.

\section{Authors' contributions}

HD participated in the study design and coordination, contributed to study selection, evaluation and grading and data extraction and interpretation, performed the statistical analysis and data meta-analysis and drafted and revised the manuscript. AA participated in the study design and coordination, contributed to study selection and evaluation and data extraction and interpretation and reviewed the draft. MF conceived of the study, participated in its design, contributed to study grading and data interpretation and reviewed the draft. MHM conceived of the study, participated in its design, contributed to study selection and data interpretation and helped to write the draft. YA conceived of the study, participated in its design, contributed to study selection, evaluation and grading and data extraction and interpretation, contributed to writing the draft and revised the manuscript. All authors read and approved the final manuscript.

\section{Competing interests}

The authors declare that they have no competing interests.

\section{Consent for publication}

Not applicable.

\section{Ethics approval and consent to participate}

Not applicable.

\section{Author details}

${ }^{1}$ College of Medicine, King Saud bin Abdulaziz University for Health Sciences, Riyadh, Saudi Arabia. ${ }^{2}$ King Abdullah International Medical Research Center, Riyadh, Saudi Arabia. ${ }^{3}$ Intensive Care Department, King Abdulaziz Medical City, P.O. Box 22490, Riyadh 11426, Saudi Arabia. ${ }^{4}$ Prince Sultan Military Medical City, Riyadh, Saudi Arabia. ${ }^{5}$ Department of Family Medicine, King Abdulaziz Medical City, Riyadh, Saudi Arabia. ${ }^{6}$ National \& Gulf Center for Evidence Based Health Practice, Riyadh 11426, Saudi Arabia. ${ }^{7}$ Center for Science of Healthcare Delivery, Mayo Clinic, Rochester, MN, USA. ${ }^{8}$ Preventive Medicine, Mayo Clinic, 200 1st Street SW, Rochester, MN 55905, USA.

Received: 16 June 2016 Accepted: 20 October 2016

Published online: 04 November 2016

References

1. Heyland DK, Dhaliwal R, Drover JW, Gramlich L, Dodek P. Canadian clinical practice guidelines for nutrition support in mechanically ventilated, critically ill adult patients. JPEN J Parenter Enteral Nutr. 2003;27(5):355-73.

2. Marik PE, Zaloga GP. Early enteral nutrition in acutely ill patients: a systematic review. Crit Care Med. 2001;29(12):2264-70.

3. Taylor SJ, Fettes SB, Jewkes C, Nelson RJ. Prospective, randomized, controlled trial to determine the effect of early enhanced enteral nutrition on clinical outcome in mechanically ventilated patients suffering head injury. Crit Care Med. 1999;27(11):2525-31.

4. Alberda C, Gramlich L, Jones N, Jeejeebhoy K, Day AG, Dhaliwal R, Heyland DK The relationship between nutritional intake and clinical outcomes in critically ill patients: results of an international multicenter observational study. Intensive Care Med. 2009;35(10):1728-37.

5. Crosara IC, Melot C, Preiser JC. A J-shaped relationship between caloric intake and survival in critically ill patients. Ann Intensive Care. 2015;5(1):37.

6. Weijs PJ, Stapel SN, de Groot SD, Driessen RH, de Jong E, Girbes AR, van Schijndel RJS, Beishuizen A. Optimal protein and energy nutrition decreases mortality in mechanically ventilated, critically ill patients: a prospective observational cohort study. JPEN J Parenter Enteral Nutr. 2012;36(1):60-8.

7. Heyland DK, Cahill NE, Dhaliwal R, Sun X, Day AG, McClave SA. Impact of enteral feeding protocols on enteral nutrition delivery: results of a multicenter observational study. JPEN J Parenter Enteral Nutr. 2010;34(6):675-84. 
8. Taylor BE, McClave SA, Martindale RG, Warren MM, Johnson DR, Braunschweig C, McCarthy MS, Davanos E, Rice TW, Cresci GA, et al. Guidelines for the Provision and Assessment of Nutrition Support Therapy in the Adult Critically III Patient: Society of Critical Care Medicine (SCCM) and American Society for Parenteral and Enteral Nutrition (A.S.P.E.N.). Crit Care Med. 2016:44(2):390-438.

9. Martin CM, Doig GS, Heyland DK, Morrison T, Sibbald WJ. Southwestern Ontario Critical Care Research N. Multicentre, cluster-randomized clinical trial of algorithms for critical-care enteral and parenteral therapy (ACCEPT). CMAJ. 2004;170(2):197-204.

10. Doig GS, Simpson F, Finfer S, Delaney A, Davies AR, Mitchell I, Dobb G. Nutrition Guidelines Investigators of the ACTG. Effect of evidence-based feeding guidelines on mortality of critically ill adults: a cluster randomized controlled trial. JAMA. 2008;300(23):2731-41.

11. Deane AM, Dhaliwal R, Day AG, Ridley EJ, Davies AR, Heyland DK. Comparisons between intragastric and small intestinal delivery of enteral nutrition in the critically ill: a systematic review and meta-analysis. Crit Care. 2013;17(3):R125.

12. Krishnan JA, Parce PB, Martinez A, Diette GB, Brower RG. Caloric intake in medical ICU patients: consistency of care with guidelines and relationship to clinical outcomes. Chest. 2003;124(1):297-305.

13. Dickerson RN. Optimal caloric intake for critically ill patients: first, do no harm. Nutr Clin Pract. 2011;26(1):48-54.

14. Arabi YM, Tamim HM, Dhar GS, Al-Dawood A, Al-Sultan M, Sakkijha MH, Kahoul SH, Brits R. Permissive underfeeding and intensive insulin therapy in critically ill patients: a randomized controlled trial. Am J Clin Nutr. 2011;93(3):569-77.

15. Rice TW, Mogan S, Hays MA, Bernard GR, Jensen GL, Wheeler AP. Randomized trial of initial trophic versus full-energy enteral nutrition in mechanically ventilated patients with acute respiratory failure. Crit Care Med. 2011;39(5):967-74.

16. Rice TW, Wheeler AP, Thompson BT, Steingrub J, Hite RD, Moss M, Morris A, Dong N, Rock P. Initial trophic vs full enteral feeding in patients with acute lung injury: the EDEN randomized trial. JAMA. 2012;307(8):795-803.

17. Ahrens CL, Barletta JF, Kanji S, Tyburski JG, Wilson RF, Janisse JJ, Devlin JW Effect of low-calorie parenteral nutrition on the incidence and severity of hyperglycemia in surgical patients: a randomized, controlled trial. Crit Care Med. 2005;33(11):2507-12.

18. McClave SA, Taylor BE, Martindale RG, Warren MM, Johnson DR, Braunschweig C, McCarthy MS, Davanos E, Rice TW, Cresci GA, et al. Guidelines for the Provision and Assessment of Nutrition Support Therapy in the Adult Critically III Patient: Society of Critical Care Medicine (SCCM) and American Society for Parenteral and Enteral Nutrition (A.S.P.E.N.). JPEN J Parenter Enteral Nutr. 2016;40(2):159-211.

19. Heyland DK, Cahill N, Day AG. Optimal amount of calories for critically ill patients: depends on how you slice the cake! Crit Care Med. 2011;39(12):2619-26.

20. Preiser JC, van Zanten AR, Berger MM, Biolo G, Casaer MP, Doig GS, Griffiths RD, Heyland DK, Hiesmayr M, lapichino G, et al. Metabolic and nutritional support of critically ill patients: consensus and controversies. Crit Care. 2015;19:35.

21. Moher D, Liberati A, Tetzlaff J, Altman DG. Preferred reporting items for systematic reviews and meta-analyses: the PRISMA statement. Ann Intern Med. 2009:151(4):264-9. W264.

22. Charles EJ, Petroze RT, Metzger R, Hranjec T, Rosenberger LH, Riccio LM, McLeod MD, Guidry CA, Stukenborg GJ, Swenson BR, et al. Hypocaloric compared with eucaloric nutritional support and its effect on infection rates in a surgical intensive care unit: a randomized controlled trial. Am J Clin Nutr. 2014;100(5):1337-43.

23. Rugeles SJ, Rueda JD, Diaz CE, Rosselli D. Hyperproteic hypocaloric enteral nutrition in the critically ill patient: a randomized controlled clinical trial. Indian J Crit Care Med. 2013;17(6):343-9.

24. Higgins JP, Altman DG, Gotzsche PC, Juni P, Moher D, Oxman AD, Savovic J, Schulz KF, Weeks L, Sterne JA. The Cochrane Collaboration's tool for assessing risk of bias in randomised trials. BMJ. 2011;343:d5928.

25. DerSimonian R, Laird N. Meta-analysis in clinical trials. Control Clin Trials. 1986;7(3):177-88.

26. Higgins JP, Thompson SG, Deeks JJ, Altman DG. Measuring inconsistency in meta-analyses. BMJ. 2003;327(7414):557-60.

27. Pfab F, Winhard M, Nowak-Machen M, Napadow V, Irnich D, Pawlik M, Bein T, Hansen E. Acupuncture in critically ill patients improves delayed gastric emptying: a randomized controlled trial. Anesth Analg. 2011;112(1):150-5.
28. Kaur N, Gupta MK, Minocha VR. Early enteral feeding by nasoenteric tubes in patients with perforation peritonitis. World J Surg. 2005;29(8):1023-7. discussion 1027-1028.

29. Berne JD, Norwood SH, McAuley CE, Vallina VL, Villareal D, Weston J, McClarty J. Erythromycin reduces delayed gastric emptying in critically ill trauma patients: a randomized, controlled trial. J Trauma. 2002;53(3):422-5.

30. Nguyen NQ, Chapman MJ, Fraser RJ, Bryant LK, Holloway RH. Erythromycin is more effective than metoclopramide in the treatment of feed intolerance in critical illness. Crit Care Med. 2007;35(2):483-9.

31. Seder CW, Stockdale W, Hale L, Janczyk RJ. Nasal bridling decreases feeding tube dislodgment and may increase caloric intake in the surgical intensive care unit: a randomized, controlled trial. Crit Care Med. 2010;38(3):797-801.

32. Chapman MJ, Fraser RJ, Kluger MT, Buist MD, De Nichilo DJ. Erythromycin improves gastric emptying in critically ill patients intolerant of nasogastric feeding. Crit Care Med. 2000;28(7):2334-7.

33. Taylor SJ. Early enhanced enteral nutrition in burned patients is associated with fewer infective complications and shorter hospital stay. J Hum Nutr Diet. 1999;12:85-91.

34. Spapen HD, Duinslaeger L, Diltoer M, Gillet R, Bossuyt A, Huyghens LP. Gastric emptying in critically ill patients is accelerated by adding cisapride to a standard enteral feeding protocol: results of a prospective, randomized, controlled trial. Crit Care Med. 1995;23(3):481-5.

35. Boivin MA, Levy H. Gastric feeding with erythromycin is equivalent to transpyloric feeding in the critically ill. Crit Care Med. 2001;29(10):1916-9.

36. Davies AR, Froomes PR, French CJ, Bellomo R, Gutteridge GA, Nyulasi I, Walker R, Sewell RB. Randomized comparison of nasojejunal and nasogastric feeding in critically ill patients. Crit Care Med. 2002;30(3):586-90.

37. Neumann DA, DeLegge MH. Gastric versus small-bowel tube feeding in the intensive care unit: a prospective comparison of efficacy. Crit Care Med. 2002;30(7):1436-8.

38. MacLeod JB, Lefton J, Houghton D, Roland C, Doherty J, Cohn SM, Barquist ES. Prospective randomized control trial of intermittent versus continuous gastric feeds for critically ill trauma patients. J Trauma. 2007;63(1):57-61.

39. Montejo JC, Grau T, Acosta J, Ruiz-Santana S, Planas M, Garcia-De-Lorenzo A, Mesejo A, Cervera M, Sanchez-Alvarez C, Nunez-Ruiz R, et al. Multicenter, prospective, randomized, single-blind study comparing the efficacy and gastrointestinal complications of early jejunal feeding with early gastric feeding in critically ill patients. Crit Care Med. 2002:30(4):796-800.

40. Day L, Stotts NA, Frankfurt A, Stralovich-Romani A, Volz M, Muwaswes M, Fukuoka Y, O'Leary-Kelley C. Gastric versus duodenal feeding in patients with neurological disease: a pilot study. J Neurosci Nurs. 2001;33(3):148-9. 155-149.

41. Kortbeek JB, Haigh PI, Doig C. Duodenal versus gastric feeding in ventilated blunt trauma patients: a randomized controlled trial. J Trauma. 1999;46(6):992-6. discussion 996-998.

42. Gottschlich MM, Jenkins M, Warden GD, Baumer T, Havens P, Snook JT, Alexander JW. Differential effects of three enteral dietary regimens on selected outcome variables in burn patients. JPEN J Parenter Enteral Nutr. 1990;14(3):225-36.

43. Garrel DR, Razi M, Lariviere F, Jobin N, Naman N, Emptoz-Bonneton A, Pugeat MM. Improved clinical status and length of care with low-fat nutrition support in burn patients. JPEN J Parenter Enteral Nutr. 1995;19(6):482-91.

44. Tamion F, Hamelin K, Duflo A, Girault C, Richard JC, Bonmarchand G. Gastric emptying in mechanically ventilated critically ill patients: effect of neuromuscular blocking agent. Intensive Care Med. 2003;29(10):1717-22.

45. Liu Z, Su L, Liao Y, Liu J. Impact of target energy intake on outcomes in septic patients: a prospective randomized controlled trial. Zhonghua Wei Zhong Bing Ji Jiu Yi Xue. 2014;26(3):131-4.

46. Kadamani I, Itani M, Zahran E, Taha N. Incidence of aspiration and gastrointestinal complications in critically ill patients using continuous versus bolus infusion of enteral nutrition: a pseudo-randomised controlled trial. Aust Crit Care. 2014;27(4):188-93.

47. Uysal N, Eser I, Akpinar H. The effect of abdominal massage on gastric residual volume: a randomized controlled trial. Gastroenterol Nurs. 2012;35(2):117-23.

48. Wills AM, Hubbard J, Macklin EA, Glass J, Tandan R, Simpson EP, Brooks B, Gelinas D, Mitsumoto H, Mozaffar T, et al. Hypercaloric enteral nutrition in patients with amyotrophic lateral sclerosis: a randomised, double-blind, placebo-controlled phase 2 trial. Lancet. 2014;383(9934):2065-72.

49. Heyland DK, Murch L, Cahill N, McCall M, Muscedere J, Stelfox HT, Bray T, Tanguay $T$, Jiang $X$, Day AG. Enhanced protein-energy provision via the enteral route feeding protocol in critically ill patients: results of a cluster randomized trial. Crit Care Med. 2013;41(12):2743-53. 
50. Li YL, Ma L, Fan XQ. Clinical effects of early standardized enteral nutrition through a nasogastric tube combined with effective monitoring in patients with gastric cancer. [Chinese]. World Chinese J Digestol. 2015;23(21):3446-50.

51. McClave SA, Saad MA, Esterle M, Anderson M, Jotautas AE, Franklin GA, Heyland DK, Hurt RT. Volume-based feeding in the critically ill patient. JPEN J Parenter Enteral Nutr. 2015:39(6):707-12.

52. Wan B, Fu H, Yin J. Early jejunal feeding by bedside placement of a nasointestinal tube significantly improves nutritional status and reduces complications in critically ill patients versus enteral nutrition by a nasogastric tube. Asia Pac J Clin Nutr. 2015;24(1):51-7.

53. Petros $\mathrm{S}$, Horbach M, Seidel F, Weidhase L. Hypocaloric vs normocaloric nutrition in critically ill patients: a prospective randomized pilot trial. JPEN J Parenter Enteral Nutr. 2016;40(2):242-9.

54. Montecalvo MA, Steger KA, Farber HW, Smith BF, Dennis RC, Fitzpatrick GF, Pollack SD, Korsberg TZ, Birkett DH, Hirsch EF, et al. Nutritional outcome and pneumonia in critical care patients randomized to gastric versus jejunal tube feedings. The Critical Care Research Team. Crit Care Med. 1992;20(10):1377-87.

55. Kearns PJ, Chin D, Mueller L, Wallace K, Jensen WA, Kirsch CM. The incidence of ventilator-associated pneumonia and success in nutrient delivery with gastric versus small intestinal feeding: a randomized clinical trial. Crit Care Med. 2000;28(6):1742-6.

56. Chen YC, Chou SS, Lin LH, Wu LF. The effect of intermittent nasogastric feeding on preventing aspiration pneumonia in ventilated critically ill patients. J Nurs Res. 2006;14(3):167-80.

57. Nguyen NQ, Chapman M, Fraser RJ, Bryant LK, Burgstad C, Holloway RH. Prokinetic therapy for feed intolerance in critical illness: one drug or two? Crit Care Med. 2007;35(11):2561-7.

58. Desachy A, Clavel M, Vuagnat A, Normand S, Gissot V, Francois B. Initial efficacy and tolerability of early enteral nutrition with immediate or gradual introduction in intubated patients. Intensive Care Med. 2008;34(6):1054-9.

59. Hsu CW, Sun SF, Lin SL, Kang SP, Chu KA, Lin CH, Huang HH. Duodenal versus gastric feeding in medical intensive care unit patients: a prospective, randomized, clinical study. Crit Care Med. 2009;37(6):1866-72.

60. White H, Sosnowski K, Tran K, Reeves A, Jones M. A randomised controlled comparison of early post-pyloric versus early gastric feeding to meet nutritional targets in ventilated intensive care patients. Crit Care. 2009;13(6):R187.

61. Montejo JC, Minambres E, Bordeje L, Mesejo A, Acosta J, Heras A, Ferre M, Fernandez-Ortega F, Vaquerizo Cl, Manzanedo R. Gastric residual volume during enteral nutrition in ICU patients: the REGANE study. Intensive Care Med. 2010;36(8):1386-93.

62. Acosta-Escribano J, Fernandez-Vivas M, Grau Carmona T, Caturla-Such J, Garcia-Martinez M, Menendez-Mainer A, Solera-Suarez M, Sanchez-Paya J. Gastric versus transpyloric feeding in severe traumatic brain injury: a prospective, randomized trial. Intensive Care Med. 2010;36(9):1532-9.

63. Singer P, Anbar R, Cohen J, Shapiro H, Shalita-Chesner M, Lev S, Grozovski E, Theilla M, Frishman S, Madar Z. The tight calorie control study (TICACOS): a prospective, randomized, controlled pilot study of nutritional support in critically ill patients. Intensive Care Med. 2011;37(4):601-9.

64. Huang HH, Chang SJ, Hsu CW, Chang TM, Kang SP, Liu MY. Severity of illness influences the efficacy of enteral feeding route on clinical outcomes in patients with critical illness. J Acad Nutr Diet. 2012;112(8):1138-46.

65. Reignier J, Mercier E, Le Gouge A, Boulain T, Desachy A, Bellec F, Clavel M, Frat JP, Plantefeve G, Quenot JP, et al. Effect of not monitoring residual gastric volume on risk of ventilator-associated pneumonia in adults receiving mechanical ventilation and early enteral feeding: a randomized controlled trial. JAMA. 2013;309(3):249-56.

66. Peake SL, Davies AR, Deane AM, Lange K, Moran JL, O'Connor SN, Ridley EJ, Williams PJ, Chapman MJ, investigators T, et al. Use of a concentrated enteral nutrition solution to increase calorie delivery to critically ill patients: a randomized, double-blind, clinical trial. Am J Clin Nutr. 2014;100(2):616-25.

67. Braunschweig CA, Sheean PM, Peterson SJ, Gomez Perez S, Freels S, Lateef O, Gurka D, Fantuzzi G. Intensive nutrition in acute lung injury: a clinical trial (INTACT). JPEN J Parenter Enteral Nutr. 2015;39(1):13-20.

68. Arabi YM, Aldawood AS, Haddad SH, Al-Dorzi HM, Tamim HM, Jones G, Mehta S, Mclntyre L, Solaiman O, Sakkijha MH, et al. Permissive underfeeding or standard enteral feeding in critically ill adults. N Engl J Med. 2015;372(25):2398-408.

69. Doig GS, Simpson F, Heighes PT, Bellomo R, Chesher D, Caterson ID, Reade MC, Harrigan PW. Refeeding Syndrome Trial Investigators G. Restricted versus continued standard caloric intake during the management of refeeding syndrome in critically ill adults: a randomised, parallel-group, multicentre, singleblind controlled trial. Lancet Respir Med. 2015;3(12):943-52.
70. McClave SA, Lowen CC, Kleber MJ, Nicholson JF, Jimmerson SC, McConnell JW, Jung LY. Are patients fed appropriately according to their caloric requirements? JPEN J Parenter Enteral Nutr. 1998;22(6):375-81.

71. Kolditz Cl, Langin D. Adipose tissue lipolysis. Curr Opin Clin Nutr Metab Care. 2010;13(4):377-81.

72. Chiolero R, Revelly JP, Tappy L. Energy metabolism in sepsis and injury. Nutrition. 1997;13(9 Suppl):45S-51S.

73. Mailloux RJ, Harper ME. Uncoupling proteins and the control of mitochondrial reactive oxygen species production. Free Radic Biol Med. 2011;51(6):1106-15.

74. Gunst J, Derese I, Aertgeerts A, Ververs EJ, Wauters A, Van den Berghe G, Vanhorebeek I. Insufficient autophagy contributes to mitochondrial dysfunction, organ failure, and adverse outcome in an animal model of critical illness. Crit Care Med. 2013;41(1):182-94.

75. Vanhorebeek I, Gunst J, Derde S, Derese I, Boussemaere M, Guiza F, Martinet W, Timmermans JP, D'Hoore A, Wouters PJ, et al. Insufficient activation of autophagy allows cellular damage to accumulate in critically ill patients. J Clin Endocrinol Metab. 2011;96(4):E633-645.

76. Marik PE, Hooper MH. Normocaloric versus hypocaloric feeding on the outcomes of ICU patients: a systematic review and meta-analysis. Intensive Care Med. 2016:42(3):316-23.

77. Heyland DK, Dhaliwal R, Lemieux M. More questions than answers. JPEN J Parenter Enteral Nutr. 2015:39(2):143.

78. Berger MM, Pichard C. Understanding the causes of death in INTACT by Braunschweig et al. JPEN J Parenter Enteral Nutr. 2015;39(2):144.

79. Braunschweig C, Sheean P, Peterson SJ, Perez SG, Freels S, Lateef O, Gurka D, Fantuzzi G. Response to Berger and Pichard and Heyland et al. JPEN J Parenter Enteral Nutr. 2015;39(2):144-5.

80. Gramlich L, Kichian K, Pinilla J, Rodych NJ, Dhaliwal R, Heyland DK. Does enteral nutrition compared to parenteral nutrition result in better outcomes in critically ill adult patients? A systematic review of the literature. Nutrition. 2004;20(10):843-8.

81. Lewis SJ, Egger M, Sylvester PA, Thomas S. Early enteral feeding versus "nil by mouth" after gastrointestinal surgery: systematic review and meta-analysis of controlled trials. BMJ. 2001;323(7316):773-6.

82. Lempiainen J, Finckenberg P, Mervaala EE, Sankari S, Levijoki J, Mervaala EM. Caloric restriction ameliorates kidney ischaemia/reperfusion injury through PGC-1alpha-eNOS pathway and enhanced autophagy. Acta Physiol (Oxf). 2013;208(4):410-21.

83. Ning YC, Cai GY, Zhuo L, Gao JJ, Dong D, Cui SY, Shi SZ, Feng Z, Zhang L, Sun XF, et al. Beneficial effects of short-term calorie restriction against cisplatin-induced acute renal injury in aged rats. Nephron Exp Nephrol. 2013;124(3-4):19-27.

84. Mitchell JR, Verweij M, Brand K, van de Ven M, Goemaere N, van den Engel S, Chu T, Forrer $F$, Muller $C$, de Jong $M$, et al. Short-term dietary restriction and fasting precondition against ischemia reperfusion injury in mice. Aging Cell. 2010;9(1):40-53.

85. Bellomo R, Cass A, Cole L, Finfer S, Gallagher M, Lee J, Lo S, McArthur C, McGuinness S, Myburgh J, et al. Calorie intake and patient outcomes in severe acute kidney injury: findings from The Randomized Evaluation of Normal vs. Augmented Level of Replacement Therapy (RENAL) study trial. Crit Care. 2014;18(2):R45.

86. Villet S, Chiolero RL, Bollmann MD, Revelly JP, Cayeux RNM, Delarue J, Berger MM. Negative impact of hypocaloric feeding and energy balance on clinical outcome in ICU patients. Clin Nutr. 2005;24(4):502-9.

87. Dickerson RN, Boschert KJ, Kudsk KA, Brown RO. Hypocaloric enteral tube feeding in critically ill obese patients. Nutrition. 2002;18(3):241-6.

88. Ibrahim EH, Mehringer L, Prentice D, Sherman G, Schaiff R, Fraser V, Kollef MH. Early versus late enteral feeding of mechanically ventilated patients: results of a clinical trial. JPEN J Parenter Enteral Nutr. 2002;26(3):174-81.

89. Heidegger CP, Berger MM, Graf S, Zingg W, Darmon P, Costanza MC, Thibault R, Pichard C. Optimisation of energy provision with supplemental parenteral nutrition in critically ill patients: a randomised controlled clinical trial. Lancet. 2013;381(9864):385-93.

90. Harvey SE, Parrott F, Harrison DA, Bear DE, Segaran E, Beale R, Bellingan G, Leonard R, Mythen MG, Rowan KM, et al. Trial of the route of early nutritional support in critically ill adults. N Engl J Med. 2014;371(18):1673-84.

91. Jiang H, Sun MW, Hefright B, Chen W, Lu CD, Zeng J. Efficacy of hypocaloric parenteral nutrition for surgical patients: a systematic review and metaanalysis. Clin Nutr. 2011;30(6):730-7.

92. De Waele E, Opsomer T, Honore PM, Diltoer M, Mattens S, Huyghens L, Spapen $\mathrm{H}$. Measured versus calculated resting energy expenditure in critically ill adult patients. Do mathematics match the gold standard? Minerva Anestesiol. 2015;81(3):272-82. 